\title{
The Chromatin Remodeling Protein Bptf Promotes Posterior Neuroectodermal Fate by Enhancing Smad2-Activated wnt8a Expression
}

\author{
Yuanqing Ma, ${ }^{1}$ Xiuli Liu, ${ }^{1}$ Zhaoting Liu, ${ }^{1}$ Shi Wei, ${ }^{1}$ Hanqiao Shang, ${ }^{1}$ Yu Xue, ${ }^{2}$ Yu Cao, ${ }^{1}$ Anming Meng, ${ }^{2}$ \\ and Qiang Wang ${ }^{1}$ \\ ${ }^{1}$ State Key Laboratory of Biomembrane and Membrane Biotechnology, Institute of Zoology, Chinese Academy of Sciences, Beijing, China, and ${ }^{2}$ Tsinghua- \\ Peking Center for Life Sciences, School of Life Sciences, Tsinghua University, Beijing, China
}

\begin{abstract}
During vertebrate embryogenesis, the neuroectoderm is induced from dorsal ectoderm and then partitioned into anterior and posterior neuroectodermal domains by posteriorizing signals, such as Wnt and fibroblast growth factor. However, little is known about epigenetic regulation of posteriorizing gene expression. Here, we report a requirement of the chromatin remodeling protein Bptf for neuroectodermal posteriorization in zebrafish embryos. Knockdown of $b p t f$ leads to an expansion of the anterior neuroectoderm at the expense of the posterior ectoderm. Bptf functionally and physically interacts with p-Smad2, which is activated by non-Nodal TGF- $\beta$ signaling, to promote the expression of $w n t 8 a$, a critical gene for neural posteriorization. Bptf and Smad2 directly bind to and activate the wnt8a promoter through recruiting NURF remodeling complex. When $b p t f$ function or TGF- $\beta$ signal transduction is inhibited, the nucleosome density on the $w n t 8 a$ promoter is increased. We propose that Bptf and TGF- $\beta / S \operatorname{Smad} 2$ mediate nucleosome remodeling to regulate $w n t 8 a$ expression and hence neural posteriorization.
\end{abstract}

Key words: bptf; neural posteriorization; nucleosome remodeling; smad2; wnt8a

\section{Introduction}

In vertebrates, the early development of the CNS during gastrulation is accomplished via a two-step mechanism. As the first step, the neuroectoderm with anterior character is induced from the dorsal ectoderm by antagonists of bone morphogenetic proteins, such as Chordin, Noggin, and Follistatin, which act to prevent the ventrally derived bone morphogenetic proteins to use the default neural fate (Lamb et al., 1993; Hemmati-Brivanlou et al., 1994; Sasai et al., 1995; Hammerschmidt et al., 1996; SchulteMerker et al., 1997; Dal-Pra et al., 2006). As the second step, graded posteriorizing signals, including Wnt, fibroblast growth factor, and retinoic acid signals, transform the newly induced neuroectoderm into the posterior neuroectoderm, which ultimately gives rise to the posterior midbrain, the hindbrain, and the spinal cord (Cox and Hemmati-Brivanlou, 1995; Sasai and De

Received Jan. 28, 2015; revised March 31, 2015; accepted April 23, 2015.

Author contributions: Q.W. designed research; Y.M., X.L., and S.W. performed research; Z.L., Y.X., and Y.C. contributed unpublished reagents/analytic tools; H.S., A.M., and Q.W. analyzed data; Y.M., A.M., and Q.W. wrote the paper.

This work was supported by National Natural Science Foundation of China Grants 31322035 and 31271532, Major Science Programs of China Grants 2011CB943904 and 2011CBA01101, and Strategic Priority Research Program of the Chinese Academy of Sciences Grant XDA01010104. We thank Professor Guohong Li (Institute of Biophysics of the Chinese Academy of Sciences) for assistance with high-resolution MNase mapping assays.

The authors declare no competing financial interests.

Correspondence should be addressed to either of the following: Dr. Qiang Wang, State Key Laboratory of Biomembrane and Membrane Biotechnology, Institute of Zoology, Chinese Academy of Sciences, Beijing, China, E-mail: qiangwang@ioz.ac.cn; or Dr. Anming Meng, Tsinghua-Peking Center for Life Sciences, School of Life Sciences, Tsinghua University, Beijing, China, E-mail: mengam@mail.tsinghua.edu.cn.

DOI:10.1523/JNEUROSCI.0377-15.2015

Copyright $\odot 2015$ the authors $\quad 0270-6474 / 15 / 358493-14 \$ 15.00 / 0$
Robertis, 1997; Niehrs, 1999; Sirotkin et al., 2000; Lekven et al., 2001; Erter et al., 2001; Rentzsch et al., 2004; Maden, 2006; Stern, 2006).

TGF- $\beta /$ nodal/activin-related factors have pivotal roles in mesendoderm induction and dorsal axis determination (Zhou et al., 1993; Feldman et al., 1998), but their function in neural posteriorization is not well established. Overexpression of Antivin in zebrafish embryos, the potent antagonist of Activin ligands, results in obvious loss of posterior neural fates, but this effect may be indirect because mesendoderm induction is also abolished (Thisse et al., 2000). Although $\operatorname{Smad} 2 / 3$ activity is required for anterior-posterior (AP) patterning of the neuroectoderm in zebrafish, cell tracing experiments showed that the neuroectoderm of Nodal-deficient embryos undergoes an apparent anterior-toposterior transformation (Erter et al., 2001; Jia et al., 2009).

Bptf is the largest subunit of the nucleosome remodeling factor (NURF) complex, which was first purified from Drosophila embryo extracts and recognized to catalyze nucleosome sliding in an ATP-dependent manner to assist transcriptional activation (Tsukiyama and Wu, 1995; Mizuguchi et al., 1997). The developmental roles of Bptf in vertebrates remain largely ambiguous. The anterior visceral endoderm and the primitive streak do not form correctly in Bptfmouse mutants, suggesting a possible role of Bptf in anterior-posterior patterning (Landry et al., 2008; Goller et al., 2008).

In this study, we find that $b p t f$ is a maternally and zygotically expressed gene during early stages of zebrafish embryonic development. Loss-of-function analyses indicate that $b p t f$ and smarcalare 
both required for neural posteriorization. Bptf interacts with endogenous Smad2, and their cooperative action under the control of non-Nodal TGF- $\beta$ signaling is essential for establishing a proper AP neural pattern. Furthermore, we identify that $w n t 8 a$ is the key downstream target of Bptf and TGF- $\beta / S \operatorname{mad} 2$. Bptf and Smad 2 bind to adjacent binding motifs and recruit other NURF components, such as Smarcal, to decrease nucleosome density in the wnt 8 a promoter. Our data indicate that Bptf- and TGF- $\beta /$ Smad2-regulated nucleosome remodeling events on cisregulatory elements of the $w n t 8 a$ promoter is indispensable for zebrafish neural posteriorization.

\section{Materials and Methods}

Zebrafish strains. Tuebingen strain of zebrafish was used to obtain wildtype embryos. Embryos were maintained in Holtfreter's solution at $28.5^{\circ} \mathrm{C}$ and staged by morphology as previously described (Kimmel et al., 1995). MZoep mutant embryos were generated by crossing homozygotic male and female oep ${ }^{t z 257 / t z 257}$ adult mutants, which were rescued by injection of oep mRNA (Gritsman et al., 1999). Homozygous p53(M214K) mutant fish line (abbreviated as $p 53^{-/-}$) carrying a loss-of-function $p 53$ point mutation was kindly provided by Prof. Jinrong Peng at College of Life Sciences, Zhejiang University.

Embryonic treatment. To inhibit TGF- $\beta$ signal transduction, embryos at 16 cell stage were treated with $50 \mu \mathrm{M}$ SB431542 under dark conditions and harvested at $75 \%$ epiboly stage for Western blot or in situ hybridization.

RNA synthesis, morpholinos, and whole-mount in situ hybridization. The mRNAs encoding $w n t 8 a$, constitutively active smad2 (casmad2), dominant-negative smad2 (dnasmad2), dominant-negative TGF- $\beta$ Receptor II $(\Delta k T \beta R I I)$, smarcal, and dominant-negative smarcal (smarca1K174R) were synthesized in vitro using the mMessage mMachine kit (Ambion). Digoxigenin-UTP-labeled antisense RNA probes were in vitro transcribed using MEGAscript Kit (Ambion) according to the manufacturer's instructions. siRNAs targeting all the isoforms from zebrafish $b p t f$ gene (gene ID: 324479 , NCBI) were designed by and purchased from GenePharma. The sequences of siRNAs were as follows: negative control, sense $5^{\prime}$-ACGUGACACGUUCGGAGAATT- $3^{\prime}$, antisense 5'-UUCUUCGAACGUGUCACGUTT-3'; siRNA1, sense 5' - UUC GGUUUCAAGCUUCGGCTT-3' ${ }^{\prime}$, antisense $5^{\prime}$ - GCCGAAGCUUGAAA CCGAATT- ${ }^{\prime}$; siRNA2, sense 5' - AACACUGGAACUGAGCACCTT-3', antisense 5' - GGUGCUCAGUUCCAGUGUUTT-3'.

Antisense morpholinos were designed by and purchased from Gene Tools and have the following sequences: $b p t f$ translation-blocking morpholino (bptf MO1, positioning around the translation start site [TSS] of $b p t f)$, 5'-GCGGCCTGCCTCGTCTCCCCCTCAT-3'; $b p t f$ splicingblocking morpholino ( $b p t f \mathrm{MO}$, targeting the splicing region between exon 1 and intron1), 5' -TCCGACGAAGCGTCCGTACCTGTGT-3'; reverse control morpholino of $b p t f \mathrm{MO} 1$ (cMO), 5' -TGTGTCCATGCCT GCGAAGCAGCCT-3'. For testing the effectiveness of bptf MO1, the expression vector $b p t f-G F P$ was generated by fusing the $23 \mathrm{bp}$ upstream flanking region and the first $27 \mathrm{bp}$ of the $b p t f$ open reading frame into a pEGFP-N1 vector. Microinjection and whole-mount in situ hybridization was performed as previously described (Jia et al., 2008).

Western blot and coimmunoprecipitation. For Western blot, we used affinity-purified anti-Smad2/3 (3102, Cell Signaling Technology), antip-Smad2 (9510S, Cell Signaling Technology), and anti- $\beta$-actin (sc-1615, Santa Cruz Biotechnology) antibodies. For coimmunoprecipitation assays to study protein-protein interaction, embryos or HEK293T cells were harvested and lysed with TNE lysis buffer (10 mM Tris-HCl, pH 7.5, $150 \mathrm{~mm} \mathrm{NaCl}, 2 \mathrm{~mm}$ EDTA, and $0.5 \%$ Nonidet P-40) containing a protease inhibitor mixture. The lysates were incubated with protein A Sepharose beads and either anti-Smad2/3 or anti-Myc (562-5, MBL) antibody at $4^{\circ} \mathrm{C}$ for $4 \mathrm{~h}$. The beads were washed four times with TNE buffer. The bound proteins were separated by SDS-PAGE and visualized by Western blot.

Antibody generation. The rabbit polyclonal anti-Bptf antibody was generated by our laboratory. An epitope corresponding to residues RGRRGRPPKAQLVQEC of zebrafish Bptf was chosen for immunization. This polyclonal antibody was affinity purified and validated for specificity by peptide competition assays. The purified antibody (concentration, $200 \mu \mathrm{g} / \mathrm{ml}$ ) was used at a dilution of 1:2000 for Western blot and 1:100 for protein coimmunoprecipitation and chromatin immunoprecipitation (ChIP) assays in this study.

Dual-luciferase reporter assays. For detection and quantification of Smad2 activity in zebrafish embryos, the Smad2-specific ARE luciferase reporter construct DNA was mixed with Renilla luciferase reporter DNA in a ratio of 10:1. Wild-type and MZoep mutant embryos were injected with $100 \mathrm{pg}$ of the DNA mixture at the one-cell stage. To inhibit TGF- $\beta$ signal transduction, the injected MZoep mutant embryos were incubated with $50 \mu \mathrm{M}$ SB431542 (S4317, Sigma) from 16-cell stage under dark conditions. For analysis of promoter activity, serial truncations of the $w n t 8 a$ promoter reporter were generated and injected into embryos together with indicated MOs and RNAs. A group of 20 embryos for each sample were harvested at $75 \%$ epiboly stage and lysed with passive lysis buffer (Promega) for detecting luciferase activities.

For the luciferase reporter assays performed in cell culture, HEK293 cells were transfected with the indicated constructs together with Renilla luciferase reporter DNA (20 ng) as the internal control. Cells were stimulated with TGF- $\beta 1$ ( $1 \mathrm{ng} / \mathrm{ml})$ or Activin A (10 ng/ml) for $16 \mathrm{~h}$ before harvest for luciferase assay.

Each experiment was performed in triplicate, and the data represent the mean $\pm \mathrm{SD}$ of three independent experiments after normalized to Renilla luciferase activity.

ChIP. Embryos were injected with indicated MOs or RNAs at one-cell stage and $\sim 400$ embryos were harvested at $75 \%$ epiboly stage for each assay. Then these embryos were crosslinked with $1.85 \%$ for $15 \mathrm{~min}$ at room temperature and lysed. DNA/protein complex-containing lysate was sonicated to an apparent length of $300-1000 \mathrm{bp}$ and then centrifuged at $14,000 \mathrm{rpm}$ for $10 \mathrm{~min}$. The indicated antibody (anti-Smad2/3 or anti-BPTF) was incubated with the diluted chromatin supernatant and BSA-blocked protein A beads overnight at $4^{\circ} \mathrm{C}$. The immunocomplex was washed sequentially with salt buffers and then eluted in elution buffer. The immunoprecipitated complexes and input fraction were reversecrosslinked by overnight incubation at $65^{\circ} \mathrm{C}$. DNA was extracted with phenol:chloroform and precipitated with ethanol after treatment with RNaseA and proteinase K. Input and immunoprecipitated DNA were subjected to PCR cycles with the following specific primers: wnt8a up forward primer, $5^{\prime}$-CTACACATTTCATACACATCGTTGA-3' (-1072 bp to $-1048 \mathrm{bp}$, upstream region of wnt8a TSS); wnt8a up reverse primer, $5^{\prime}$-TGGGAAAAGCTCTGGTGTGAAAC-3' ( -914 bp to -892 bp, upstream region of wnt8a TSS); wnt8a down forward primer, 5'-CAAGCACGGAAGTTGGAGATGGATA-3' (643 bp to $667 \mathrm{bp}$, downstream region of $w n t 8 a$ TSS); wnt8a down reverse primer, $5^{\prime}$ TTCCCGCTTTGTAGACATTCCCT-3' (820-842 bp, downstream region of $w n t 8 a$ TSS).

MNase mapping assay. Wild-type and bptf MOs or $\Delta k T \beta R I I$ mRNA injected embryos were harvested at $75 \%$ epiboly stage, and mononucleosomes were prepared using Nucleosome Preparation Kit (5333, TaKaRa) according to the manufacturer's instructions. Then the resulting mononucleosome-sized DNA samples were analyzed by qRT-PCR with overlapping primer pairs covering the wnt8a promoter region from -1449 to -416 upstream of $w n t 8 a$ TSS. Each set of primer pairs spanned across $\sim 100$ bp region and were located $30 \pm 10$ bp away from neighboring primer pairs as previously described (Li et al., 2010).

\section{Results}

\section{bptf is ubiquitously expressed during early} embryo development

We found in a previous study that the DNA-binding motif recognized by the chromatin remodeling protein Bptf coexists at a high frequency with Smad2/4-binding sites in the zebrafish genome (Liu et al., 2011). Zebrafish bptf encodes a large protein of $>2800$ amino acid residues, which shares a high sequence similarity to its human and mouse orthologs $(56.6 \%$ and $55.8 \%$, respectively). The syntenic analysis of zebrafish $b p t f$ showed that its neighbor genes are also closely linked in other species, indicating that it is the authentic ortholog of mammalian Bptf (data not 

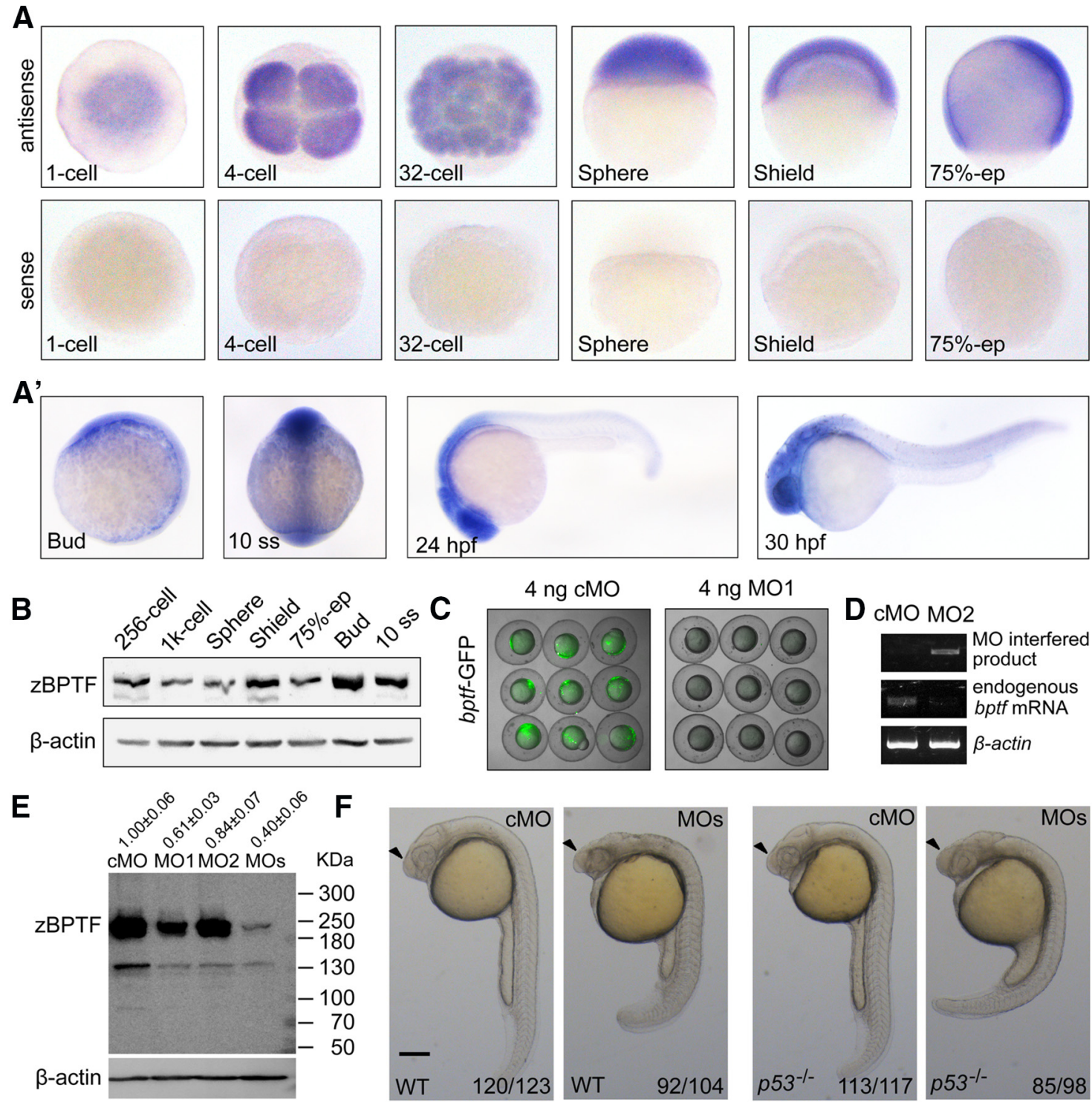

Figure 1. Zebrafish bptf is expressed during early embryonic development and involved in head patterning. $A, A^{\prime}$, Expression pattern of zebrafish bptf at indicated stages, detected by in situ hybridization with antisense $\left(\boldsymbol{A}, \boldsymbol{A}^{\prime}\right)$ or sense $(\boldsymbol{A})$ probe. From one-cell stage to 32 -cell stage, animal views; from sphere to bud stages, lateral views with dorsal to the right; 10-somite stage, dorsal view with anterior to the top; 24 and $30 \mathrm{hpf}$, lateral views with anterior to the left. $\boldsymbol{B}$, Western blot analysis of endogenous Bptf protein expression during zebrafish embryo development. $\boldsymbol{C}$, Effectiveness of bptf M01. Embryos coinjected with $50 \mathrm{pg}$ bptf-GFP plasmid DNA and $4 \mathrm{ng}$ cMO or M01 at one-cell stage. Green fluorescence was detected at $75 \%$ epiboly stage. M01-injected embryos showed a noticeable decrease in green fluorescence compared with control embryos. $\boldsymbol{D}$, Effectiveness of bptf M02. The specific splicing-interfering M0 (bptf M02, targeting the splicing region between exon 1 and intron 1 ) interfered bptf mRNA product is only detected in morphants, whereas endogenous mRNA level was significantly deceased. $\beta$-actin is used as a control. $\boldsymbol{E}$, Embryos were injected with $4 \mathrm{ng}$ bptf M01, $4 \mathrm{ng}$ bptf M02, or their mix (bptf MOs, each $4 \mathrm{ng}$ ) and harvested for Western blotting at $75 \%$ epiboly stage. UIC, Uninjected control. Band densities were analyzed using Quantity One software. The numbers on top of each lane indicate the relative band densities and the mean \pm SD of Bptf after normalization to $\beta$-actin from 4 independent experiments. $F$, Morphological effects of bptf M0-injected wild-type or $p 53$ mutant embryos at $24 \mathrm{hpf}$. Note the enlarged forebrain in bptf morphants (arrowhead). The ratio of embryos with the representative phenotypes was indicated. Scale bar, $200 \mu \mathrm{m}$.

shown). In addition, like other vertebrate orthologs, zebrafish Bptf also has several conserved domains: an N-terminal DDT domain; two plant homeodomain finger domains, one of which mediates a direct association with $\mathrm{H} 3 \mathrm{~K} 4 \mathrm{me} 3$; a glutamine-rich transcriptional activation domain; and a carboxy-terminal bromodomain that acts as an acetyl-lysine binding domain and preferentially recognizes H4K16ac (Escher et al., 2000; Zeng and Zhou, 2002; Wysocka et al., 2006; Ruthenburg et al., 2011).

We next examined the spatiotemporal expression of bptf in zebrafish embryos from one-cell stage to $30 \mathrm{~h}$ post-fertilization (hpf) by whole-mount in situ hybridization. As shown in Figure $1 A, A^{\prime}$, bptf mRNA was ubiquitously distributed before mid- segmentation stages and then retained at high levels only in the head region from $24 \mathrm{hpf}$ onward. We also generated zebrafish Bptf antibody to detect its protein expression at different stages. Western blotting revealed the existence of Bptf protein from blastula to mid-segmentation stages (Fig. 1B). These data indicates that $b p t f$ is both maternally and zygotically expressed during early embryo development.

Then we adopted knockdown approach using two antisense morpholinos: the translation blocker bptf-MO1 and the splicing blocker bptf-MO2. We found that injection of $4 \mathrm{ng} \mathrm{MO} 1$ efficiently blocked the production of the Bptf-GFP fusion protein in embryos, whereas the reverse control $\mathrm{MO}(\mathrm{cMO})$ had no effect (Fig. 1C). Furthermore, the expression of endogenous bptf 
A

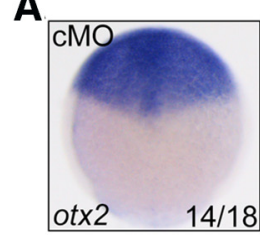

D
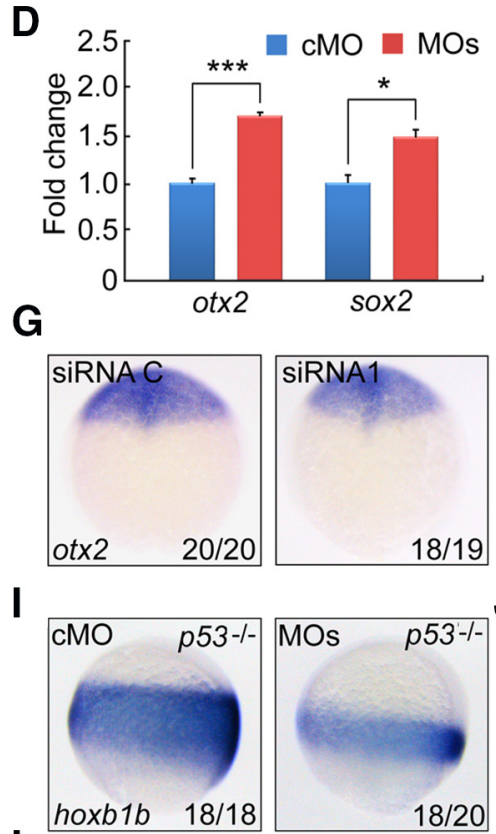

L
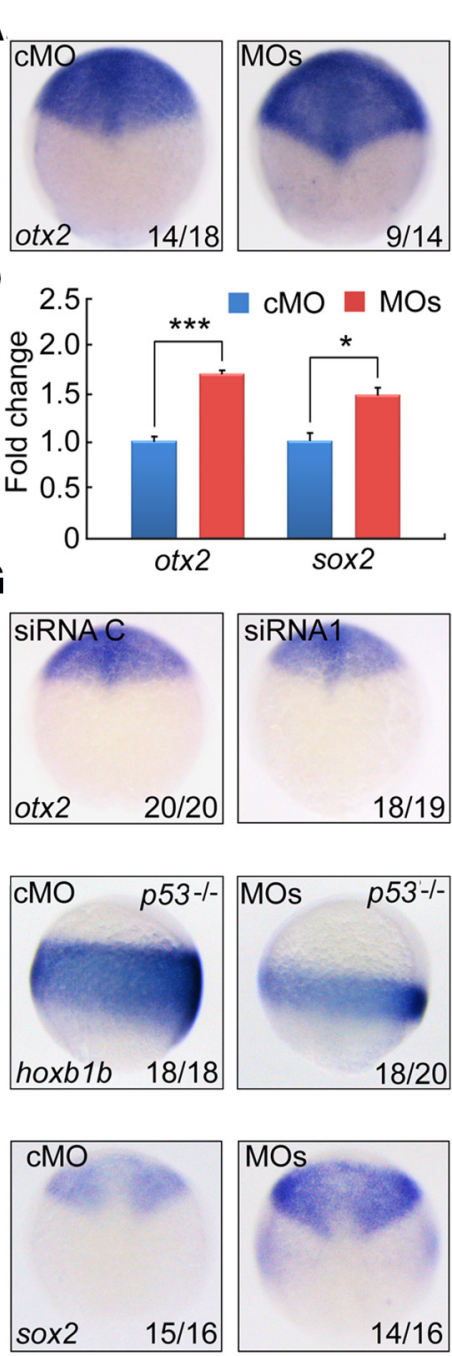

E

$E$

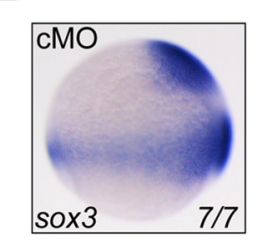

$\mathrm{H}$
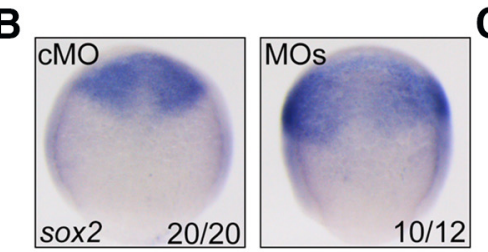

$\mathbf{F}$

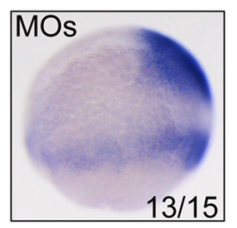

$13 / 15$
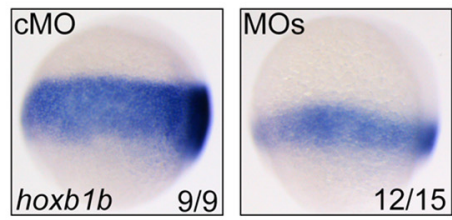

$12 / 15$

m siRNA C $\square$ siRNA1 — siRNA2
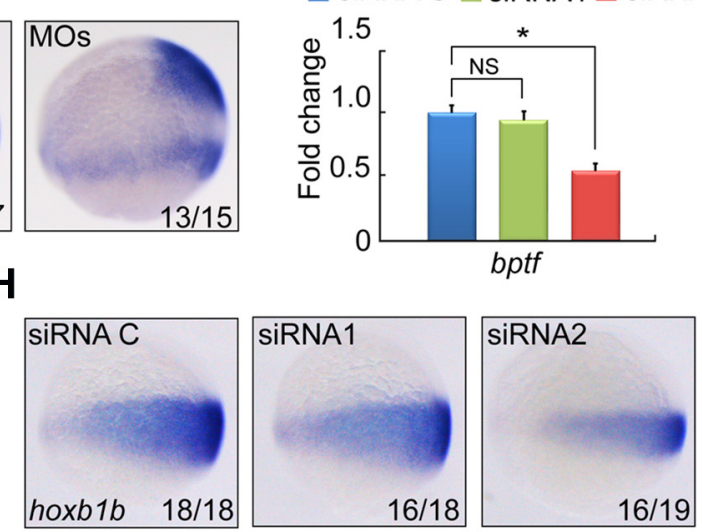

K
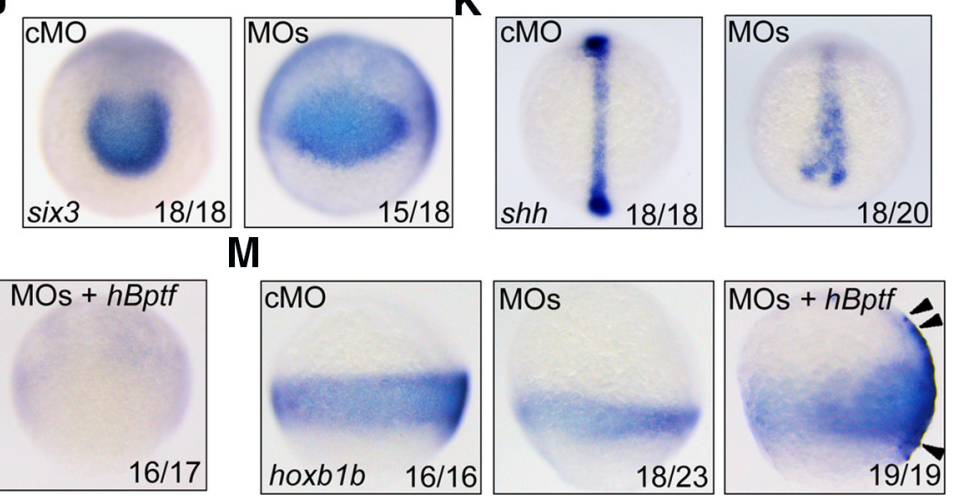

Figure 2. bptfhas crucial functions in neural posteriorization. $\boldsymbol{A}-\boldsymbol{E}$, Knockdown of bptf by morpholino injection blocks neural posteriorization. In situ hybridization of the anterior neural markers otx2 and sox2 ( $\boldsymbol{A}, \boldsymbol{B}$, dorsal view with anterior at the top), the posterior neural marker hoxb1b, and the pan-neural marker sox3 (C, $\boldsymbol{E}$, lateral views with dorsal to the right) at $75 \%$ epiboly stage. The expression of $0 t \times 2$ and sox2 in embryos injected with control M0 or bptf M0s was analyzed at $75 \%$ epiboly stage by real-time PCR. ${ }^{*} p<0.05$ (Student's $t$ test). ${ }^{* * *} p<0.001$ (Student's $t$ test). $\boldsymbol{D}$, $\boldsymbol{F}-\boldsymbol{H}$, Knockdown of bptf by siRNA injection results in severe neural patterning defects. Embryos injected with $1.2 \mathrm{ng}$ of indicated siRNAs at one-cell stage and harvested at $75 \%$ epiboly stage for real-time $P C R(\boldsymbol{F})$ and in situ hybridization $(\boldsymbol{G}, \boldsymbol{H})$ analysis. NS, Nonsignificant. ${ }^{*} p<0.05$ (Student's $t$ test). $\boldsymbol{F}, \boldsymbol{I}$, The expression pattern of hoxb $1 b$ was analyzed by in situ hybridization in control M0 and bptf M0-injected $p 53$ mutant embryos at 75\% epiboly stage. $\boldsymbol{J}, \boldsymbol{K}$, Abrogation of bptf function results in enlarged forebrain and loss of spinal cord. The expression pattern of six 3 and shh was analyzed by in situ hybridization in control M0 and bptf M0-injected embryos at the bud stage. $\boldsymbol{J}$, Anterior view with dorsal at the top. $\boldsymbol{K}, \mathrm{Dorsal}$ view, anterior is upward. $\boldsymbol{L}, \boldsymbol{M}$, Rescue of $b$ tf morphants by hBptf. The expression of sox2 $(\boldsymbol{L})$ and hoxb1b $(\boldsymbol{M})$ at $75 \%$ epiboly stage in wild-type embryos injected with bptf M0s alone or together with 50 pg plasmid construct expressing hBptf. Black arrowheads indicate the representative spotted expression of hoxb1b.

mRNA was eliminated by injection of $4 \mathrm{ng} \mathrm{MO} 2$, and the interfered mRNA product was only detected in morphants (Fig. 1D). We also examined whether the expression of endogenous Bptf protein was inhibited in morphants. Based on sequence information, the predicted molecular mass of zebrafish Bptf is $\sim 320 \mathrm{kDa}$. The Bptf antibody detected a dominant band slightly lower than expected size $(\sim 250 \mathrm{kDa})$ in wild-type zebrafish embryo lysate. Various other bands were also detected from 80 to $130 \mathrm{kDa}$ (Fig. $1 E$ ). Interestingly, all of the immunoactive bands were clearly weakened in embryo lysates from $b p t f$ morphants, suggesting the high specificity of this antibody (Fig. $1 E$ ). These faint secondary bands may represent Bptf degradation products as Bptf protein is sensitive to proteolysis (Xiao et al., 2001; Wysocka et al., 2006). More importantly, coinjection of MO1 (4 ng) and MO2 (4 ng) reduced Bptf production more efficiently (Fig. $1 E$ ). Then, coinjection of both MOs was used to knock down bptf in the subsequent experiments.
To determine the phenotypic consequences of loss of $b p t f$ function, we first examined the morphology of $b p t f$ morphants at $24 \mathrm{hpf}$. Injection bptf MOs to wild-type embryos resulted in obvious cell death in the head, a reduction in the size of the trunk and an enlargement of the forebrain (Fig. $1 F$ ). p53 mutant embryos injected with bptf MOs showed a more enlarged forebrain due to decreased cell apoptosis, excluding the possibility that the observed defects were caused by nonspecific $p 53$ activation by morpholinos (Fig. $1 F)$. These results indicate that $b p t f$ is involved in head patterning.

\section{$b p t f$ is required for zebrafish neural posteriorization}

To characterize the function of $b p t f$ during neural patterning along the AP axis, we examined the anterior neural markers ot $x 2$ and sox2, and the posterior neural marker hoxb1b respectively, at mid-gastrulation stages. As expected, $b p t f$ morphants showed a broader anterior neuroectoderm (Fig. $2 A, B$ ) and a much nar- 
rower and smaller posterior neuroectoderm (Fig. 2C). The increased expression of otx 2 and sox 2 in $b p t f$ morphants was further confirmed by RT-PCR (Fig. 2D). The expression pattern of sox3, which is expressed in both anterior and posterior neuroectoderm, was also examined in embryos at mid-gastrulation stages. The expression of sox3 in the anterior neuroectoderm was obviously expanded posteriorly, but its expression in the posterior neuroectoderm was shrunk in bptf morphants compared with control embryos (Fig. 2E). siRNA-based gene silencing has been proved to be an alternative technique for knockdown of specific gene expression in zebrafish (Dodd et al., 2004; Liu et al., 2005). To exclude the off-target effects of MO injection, we designed and injected two different siRNAs (siRNA1 and siRNA2) for targeting zebrafish bptf. As shown in Figure $2 F$, injection of siRNA2, although not siRNA1, dramatically decreased endogenous $b p t f$ expression. Embryos injected with siRNA2 exhibited obvious neural patterning defects similar to $b p t f$ morphants (Fig. $2 G, H$ ). In addition, injection bptf MOs into p53 mutant embryos also led to profound reduction of posterior neuroectoderm (Fig. 2I). Together, these results conclusively indicate that the neural patterning defects in $b p t f$ morphants are specific effects of $b p t f$ knockdown.

Analysis of more differentiated anterior and posterior neural tissues with probes for six3 (forebrain marker) and shha (spinal cord marker) transcripts revealed the enlarged forebrain and severe loss of spinal cord in $b p t f$ morphants (Fig. $2 J, K$ ). As we were unable to synthesize bptf mRNA in vitro due to its large size (nearly $9000 \mathrm{nt}$ ), 50 pg plasmid construct expressing human Bptf (hBptf) in which the MO target sequences do not exist was coinjected with MOs into embryos at one-cell stage in rescue experiments (Loosli et al., 2003). As shown in Figure $2 L, M$, the expansion of anterior neuroectoderm was repressed and the decrease of posterior neuroectoderm was obviously restored by overexpression hBptf in $b p t f$ morphants. Interestingly, because of the inhomogeneous distribution of injected plasmid DNA, spotted expression of hoxbl was observed in $h B p t f$ injected embryos (Fig. 2M). These results suggest that $b p t f$ is required for anteriorto-posterior transformation of the nascent neuroectoderm.

\section{Bptf and Smad 2 coregulate neural patterning in a Nodal- independent manner}

In the zebrafish embryo, Smad2/3 activities are demonstrated to posteriorize the neuroectoderm (Jia et al., 2009). We therefore asked whether Bptf physically and functionally interacts with Smad2 during the zebrafish neural patterning. The coimmunoprecipitation assays confirmed an association of endogenous Bptf and Smad2 in zebrafish embryos at mid-gastrulation stages (Fig. $3 A$ ). Importantly, coexpressed Bptf has much higher affinity for the phospho-mimetic Smad2(S466/468D) mutant compared with the wild-type Smad2 and phospho-resistant Smad2(S466/ 468A) mutant (Fig. 3B). Furthermore, bptf MOs-induced reduction of the hoxb1b domain could be rescued by coinjection of constitutively active smad2 (casmad2) mRNA (Fig. 3C). These results support an idea that Bptf cooperates with Smad 2 to regulate neural patterning.

Smad 2 is the intracellular mediator of TGF- $\beta$ and Nodal signaling (Massagué et al., 2005; Wu and Hill, 2009) and is activated by Nodal signaling in early vertebrate embryos to induce mesendoderm and to promote body axis patterning (Nomura and $\mathrm{Li}$, 1998; Tian and Meng, 2006; Jia et al., 2008). To determine whether Bptf and Smad2 coregulate neural patterning under the control of Nodal signaling, we first analyzed the formation of the anterior and the posterior neuroectoderm in Nodal-deficient MZoep mutants. We observed that the expression domains of both anterior (otx2 and sox2) and posterior (hoxb1b) neuroectoderm markers were decreased, but relatively well patterned alone the AP axis in mutant embryos compared with the wild-type embryos (Fig. $3 D, E$ ). We next addressed the functions of Bptf in MZoep embryos. When injected with bptf MOs, MZoep mutant embryos exhibited a marked expansion and a caudal shift of otx2 and sox 2 expression in the anterior neuroectoderm with a much smaller domain of hoxb1b in the posterior neuroectoderm (Fig. $3 D, E)$. Interestingly, a persistent expression of sox 2 was detected in the ventrolateral hoxb1b-positive cells in bptf MO-injected MZoep mutants (Fig. 3D,F). It is possible that the residual posterior neuroectodermal cells may not have been fully transformed from the anterior neural fate or these cells may be in a transitional state with bipotency for differentiation at the examined stage.

Next, we wanted to determine whether Smad2 still coregulates neural patterning with Bptf in MZoep mutants. The posterior neuroectoderm was obviously reduced by overexpression of dominant-negative smad2 (dnsmad2) in MZoep mutants (Fig. $3 H$ ), suggesting that Smad2 still plays a role in neuroectoderm posteriorization. Coinjection of casmad $2 \mathrm{mRNA}$ repressed the expansion of the anterior neuroectoderm (Fig. 3G) and restored the posterior neuroectoderm (Fig. $3 \mathrm{H}$ ) in bptf MO-injected MZoep mutants. These results imply that Bptf associates with Smad2 to promote posteriorization of the neuroectoderm in a Nodal-independent manner.

\section{Non-Nodal TGF- $\beta$ signaling plays a critical role in neural posteriorization}

SB431542 is a specific inhibitor of TGF- $\beta$ superfamily Type I activin receptor-like kinase (ALK) receptors, including ALK4, ALK5, and ALK7 (Inman et al., 2002). Embryos treated with 50 $\mu \mathrm{M}$ SB431542 from 16-cell stage to $24 \mathrm{hpf}$ were missing most of mesendodermal tissues and had fused eyes, a phenotype resembling MZoep mutants (Fig. 4A) (Gritsman et al., 1999). We then examined phosphorylated-Smad2 (p-Smad2) by Western blotting to determine whether Smad2 is activated in MZoep mutants. Result indicated that $\mathrm{p}$-Smad2 was indeed detected in MZoep mutants though much lower than in wild-type control (Fig. 4B). Importantly, the residual p-Smad2 in MZoep mutants could be largely reduced by treatment with SB431542 (Fig. 4B). The activation of Smad2 in MZoep embryos was also confirmed by Smad2-specific ARE luciferase reporter assays (Fig. 4C). These results suggest that Smad 2 is activated by non-Nodal TGF- $\beta$ signaling, which is consistent with the previous findings (Sun et al., 2006; Hagos et al., 2007).

Zebrafish $\operatorname{tg} f \beta 1 a, \operatorname{tg} f \beta 1 b$, $\operatorname{tg} f \beta 2$, and $\operatorname{tg} f \beta 3$ have been characterized (Kohli et al., 2003; Cheah et al., 2005; Cannon et al., 2013). $\operatorname{tg} f \beta 2$ and $\operatorname{tg} f \beta 3$, but not $\operatorname{tg} f \beta 1$, have been previously shown to be expressed during gastrulation (Cheah et al., 2005; Meng A, Tsinghua University, unpublished data), which raises the possibility that TGF- $\beta$ signal may have potent physiological roles in early embryonic development. Because a kinase domain truncated TGF- $\beta$ Type II receptor ( $\Delta$ kT $\beta$ RII) selectively blocks TGF- $\beta$ signaling as a dominant-negative mutant (Herskowitz, 1987; Chen et al., 1993; Brand et al., 1993), we constructed zebrafish $\Delta k T \beta R I I$ mutant plasmid by deleting the cytoplasmic serine/threonine kinase domain. Luciferase reporter assay in HEK293 cells revealed that overexpression of $\Delta k T \beta R I I$ significantly attenuated the TGF- $\beta$-induced expression of the ARE luciferase reporter (Fig. $4 D$ ), but had no obvious effects on Activin-induced luciferase activities (Fig. $4 E$ ), showing its functional specificity for inhibiting TGF- $\beta$ signaling. SB431542-treated or $\Delta k T \beta R I I$ mRNA- 
A

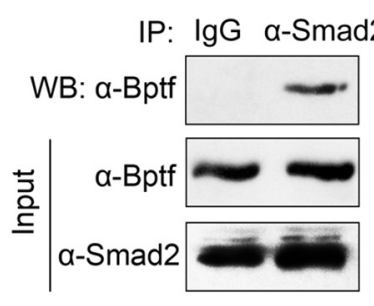

B
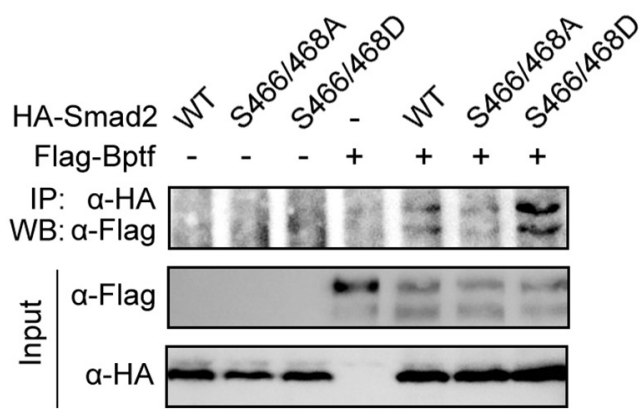

E

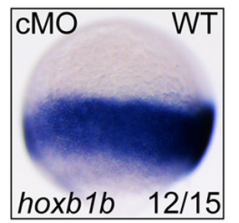

G

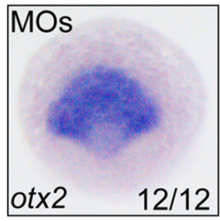

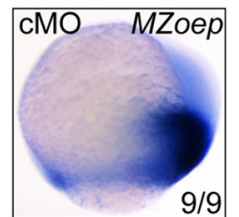

$9 / 9$

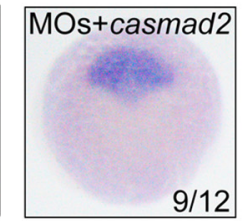

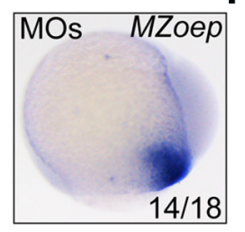

H

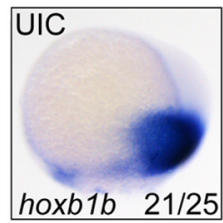

C
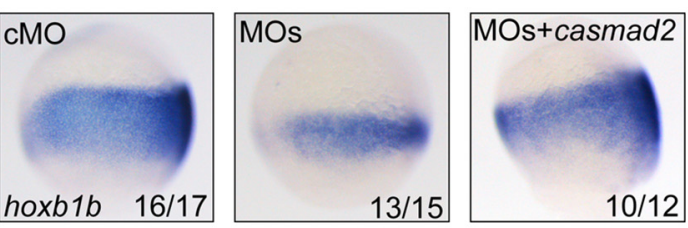

D
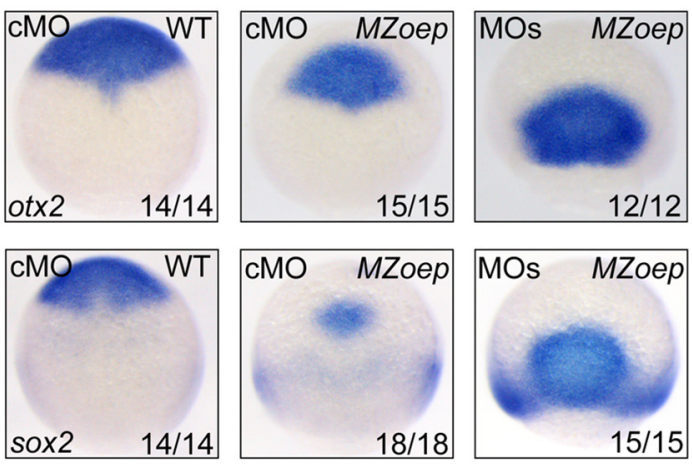

F
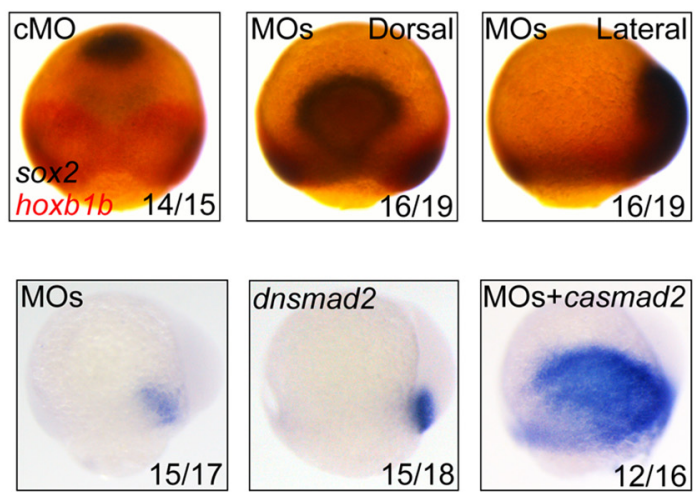

Figure 3. Bptf associates with Smad2 to posteriorize the neuroectoderm independent of Nodal signaling. $A$, Zebrafish Bptf interacts with Smad2. Embryos were harvested and lysed at the $75 \%$ epiboly stage for coimmunoprecipitation using indicated antibodies. $\boldsymbol{B}$, Bptf shows a much stronger binding affinity for activated Smad2. HEK293T cells were transfected with indicated expression plasmids encoding Flag-tagged Bptf, HA-tagged wild-type (WT) Smad2, phospho-resistant Smad2(S466/468A) mutant, and phospho-mimetic Smad2(S466/468D) mutant. Cells were harvested $48 \mathrm{~h}$ after transfection for immunoprecipitation with anti-HA antibody. C-E, Expression of neuroectodermal markers at $75 \%$ epiboly stage. Wild-type or MZoep embryos were injected with cM0 or bptfMOs alone or together with casmad $2 \mathrm{mRNA}$ at the one-cell stage and harvested later for probing with indicated probes. Embryos were shown in lateral view $(\boldsymbol{C}, \boldsymbol{E})$ or dorsal view $(\boldsymbol{D})$ with anterior to the top. $\boldsymbol{F}$, Double in situ hybridization detection of sox2 (dark blue) and hoxb1b (red) in MZoep mutants injected with cM0 or bptf MOs. The first two embryos were shown in dorsal view with anterior to the top and the last one in lateral view with dorsal to the right. $\mathbf{G}, \boldsymbol{H}$, The expression pattern of otx2 (G) and hoxb1b $(\boldsymbol{H})$ in MZoep embryos. Injection doses: cM0, $8 \mathrm{ng} ; b p t f \mathrm{MOs}, 8 \mathrm{ng}$; casmad2 mRNA, $50 \mathrm{pg}$; dnsmad2 mRNA, $600 \mathrm{pg}$. Dorsal $(\boldsymbol{G})$ or lateral views $(\boldsymbol{H})$ are shown.

injected MZoep mutants showed greatly enlarged anterior neuroectoderm and abrogated posterior neuroectoderm (Fig. $4 F-H)$. Consistently, similar results were observed in wild-type embryos injected with $\Delta k T \beta R I I$ mRNA (data not shown). Therefore, we propose that it may be canonical TGF- $\beta /$ Smad signaling that has an important function in transforming anterior neuroectoderm into posterior neuroectoderm.

\section{The lateral margin mesodermal expression of $w n t 8 a$ is regulated by $\boldsymbol{b} p t \boldsymbol{f}$ and TGF- $\beta / \mathrm{Smad} 2$}

In zebrafish, the lateral/paraxial mesodermal precursors provide crucial neural transformers, such as Wnt and fibroblast growth factor ligands, to posteriorize the nascent anterior neuroectoderm during gastrulation (Woo and Fraser, 1997; Erter et al., 2001; Lekven et al., 2001; Koshida et al., 2002). To test whether the expression of these neural transformers is regulated by bptf and TGF- $\beta / \mathrm{Smad} 2$, we analyzed the expression patterns of $f g f 3$, $f g f 8 a, w n t 3 a$, and $w n t 8 a$, which are prominently expressed during gastrulation (Buckles et al., 2004; Shimizu et al., 2005), in wildtype, MZoep mutant and $b p t f \mathrm{MO}$-injected embryos. The poten- tial downstream target genes of $b p t f$ and TGF- $\beta /$ Smad 2 during neural patterning process should meet the following conditions: expressed in lateral margin mesoderm at mid-gastrulation stages and coregulated by $b p t f$ and TGF- $\beta /$ Smad 2 signaling, although not by Nodal signaling. We observed that $f g f 3$ transcript was not expressed in the blastoderm margin at $75 \%$ epiboly stage in wildtype embryos and MZoep mutants (Fig. 5A). The expression of $f g f 8 a$ and $w n t 3 a$ was observed in the blastoderm margin at midgastrula stage, but their expression levels were not obviously downregulated in bptf morphants (Fig. $5 B, C$ ). In wild-type and $M Z$ oep mutant embryos, wnt $8 a$ showed a similar expression pattern in ventrolateral mesodermal precursors during gastrulation (Fig. $5 E$ ). Unlike $f g f 8 a$ and $w n t 3 a$, however, $w n t 8 a$ expression was abolished in bptf MO-injected wild-type and MZoep embryos (Fig. $5 D, E$ ), suggesting that its expression is regulated by $b p t f$ but not Nodal signaling. Ectopic expression of $\Delta k T \beta R I I$ effectively inhibited wnt8a expression in MZoep mutants (Fig. 5F), and overexpression of casmad 2 well restored wnt8a expression in bptf MO-injected MZoep mutants (Fig. 5G), which further supported 
A

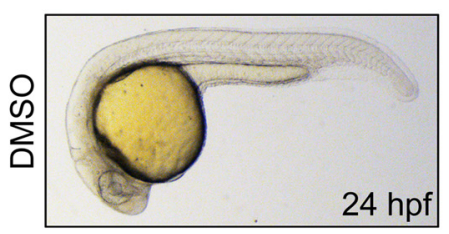

$\mathscr{\omega}$

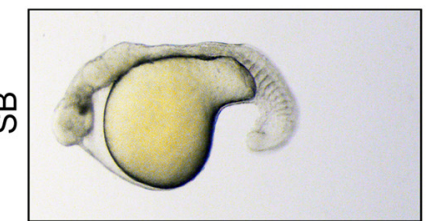

D

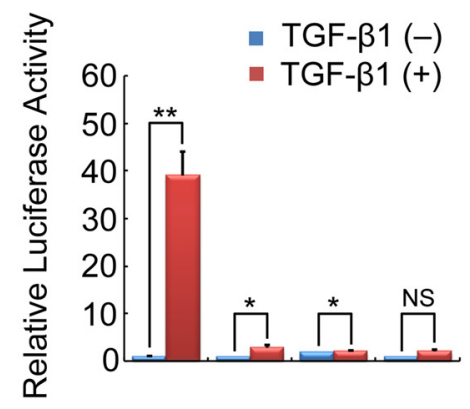

B

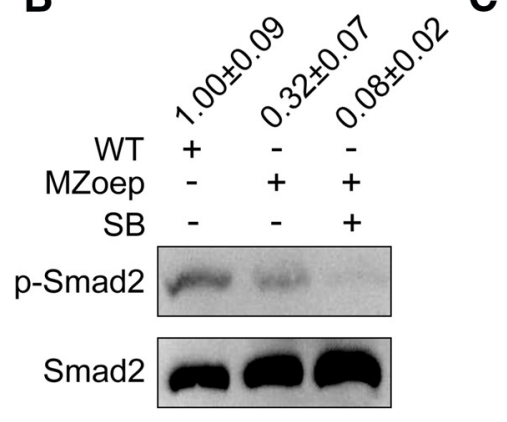

$\mathbf{E}$

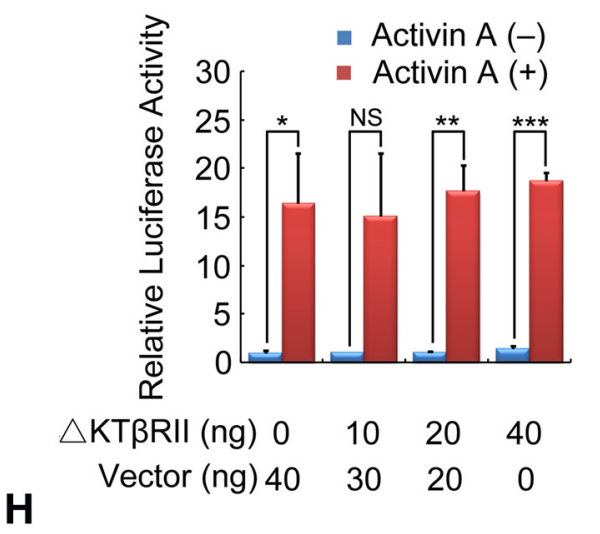

$\mathbf{F}$

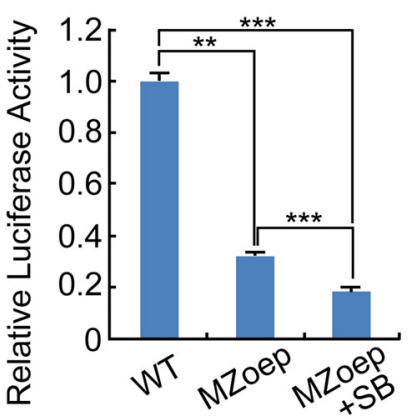

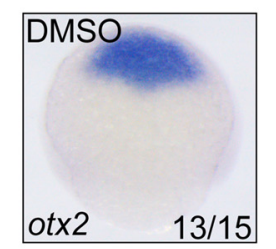
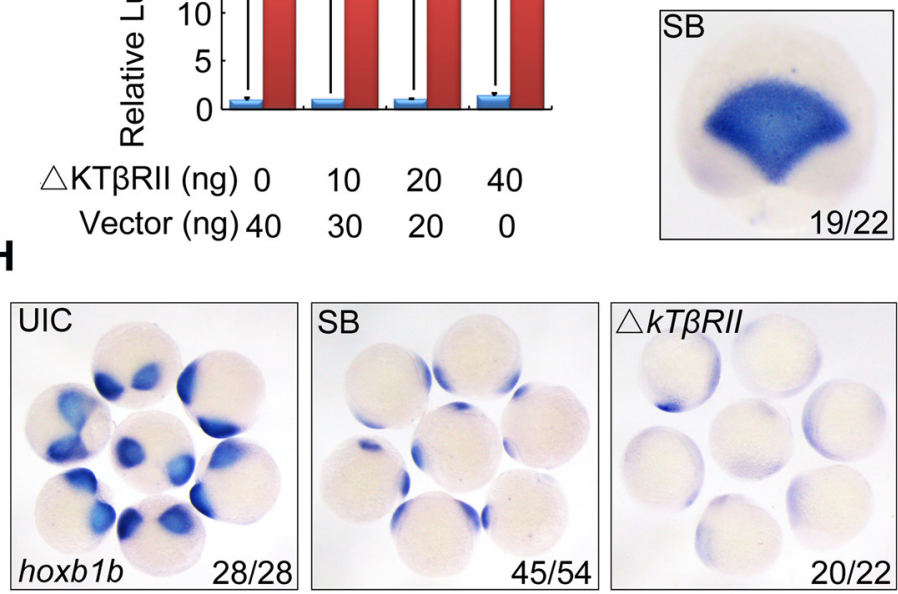

Figure 4. Inactivation of TGF- $\beta$ signaling in MZoep mutant embryos leads to severe defects in neural AP patterning. $A$, Live embryos at 24 hpf. Embryos were treated with DMSO or $50 \mu \mathrm{M}$ SB431542 (SB) from 16-cell stage to $24 \mathrm{hpf}$. The anterior is to the left. $\boldsymbol{B}$, Detection of p-Smad2 by Western blotting in wild-type and MZoep mutant embryos. Embryos (WT and MZoep) were treated with DMSO or $50 \mu \mathrm{M}$ SB431542 at 16-cell stage and harvested at 75\% epiboly stage for Western blotting. Band densities were analyzed using Quantity One software. The numbers on top of each lane indicate the relative band densities and the mean \pm SD of $\mathrm{p}$-Smad2 after normalization to $\beta$-actin from 3 independent experiments. C, Reduction of ARE-luciferase reporter expression in MZoep mutant embryos treated with SB431542. WT or MZoep embryos were injected with the reporter plasmids at one-cell stage, treated with DMSO or SB431542 at 16 -cell stage, and harvested at $75 \%$ epiboly stage for luciferase activity analysis. ${ }^{* *} p<0.01$ (Student's $t$ test). ${ }^{* * *} p<0.001$ (Student's $t$ test). $\boldsymbol{D}, \boldsymbol{E}, \Delta k T \beta R I l$ overexpression specifically attenuates TGF- $\beta$-induced expression of ARE-luciferase. HEK293 cells were cotransfected with ARE-luciferase and FoxH1 constructs along with increasing amounts of $\Delta k T \beta R / l$ construct, and treated with TGF- $\beta 1$ (1 ng/ml) (D) or Activin A $(10 \mathrm{ng} / \mathrm{ml})(\boldsymbol{E})$ for $16 \mathrm{~h}$ before harvest for luciferase assay. ${ }^{*} p<0.05$ (Student's $t$ test), ${ }^{* *} p<0.01$ (Student's $t$ test), ${ }^{* * *} p<0.001$ (Student's $t$ test); NS, nonsignificant. $\boldsymbol{F}-\boldsymbol{H}$, Change of expression pattern of otx2 and hoxb $1 b$ at the $75 \%$ epiboly stage in MZoep embryos treated with $50 \mu \mathrm{m} S B 431542$ or injected with $400 \mathrm{pg} \Delta k T \beta R / I \mathrm{mNRA}$. Single embryos were shown in dorsal view for otx2 expression $(\boldsymbol{F}, \boldsymbol{G})$ and a group of embryos were shown in dorsal view mostly with posterior to the middle for hoxb $1 b$ expression $(\boldsymbol{H})$.

an important role of TGF- $\beta /$ Smad 2 signaling for $w n t 8 a$ expression. Hence, $w n t 8 a$ is a target gene of $b p t f$ and TGF- $\beta /$ Smad2.

\section{$w n t 8 a$ acts at downstream of $b p t f$ and TGF- $\beta / S m a d 2$ to induce the posterior neural fates}

To detect whether Wnt signaling is affected by bptf knockdown during neural posteriorization, we analyzed $g f p$ reporter mRNA expression in TOPdGFP transgenic embryos, which mirrors endogenous Wnt/ $\beta$-catenin activity (Dorsky et al., 2002). Knockdown of bptf caused a drastic decrease of $d G F P$ expression in ventrolateral mesoderm from early to mid-gastrula stages (Fig. $6 A$ ), demonstrating a requirement of $b p t f$ for efficiently activating Wnt/ $\beta$-catenin signaling. We then asked whether the loss of $w n t 8 a$ expression accounted for $b p t f$ knockdown- or TGF- $\beta$ signal inhibition-induced AP patterning defects of the neuroectoderm. Coinjection of $w n t 8 a$ mRNA largely normalized ot $x 2$ and hoxblb expression in $b p t f \mathrm{MO}$-injected wild-type embryos (Fig. $6 B)$. Moreover, overexpression of $w n t 8 a$ rescued the neural patterning defects caused by coinjection of $b p t f$ MOs and $\Delta k T \beta R I I$ mRNA into wild-type embryos (Fig. 6C). Similar results were observed in further experiments using MZoep mutants (Fig. 6D). These data substantiate that function of $b p t f$ and TGF- $\beta / \operatorname{Smad} 2$ in posteriorizing the neuroectoderm is exerted by the downstream target wnt8a.

\section{Bptf and Smad 2 bind to and activate the $w n t 8 a$ promoter}

Zebrafish $w n t 8 a$ produces a bicistronic transcript, containing two open reading frames (wnt8a.1 and wnt8a.2) (Lekven et al., 2001; Ramel and Lekven, 2004). The expression of $w n t 8 a$ transcripts in ventrolateral blastodermal margin is first detected at the $30 \%$ epiboly stage and maintained during gastrulation (Kelly et al., 1995; Ramel and Lekven, 2004). To further investigate the tran- 
A

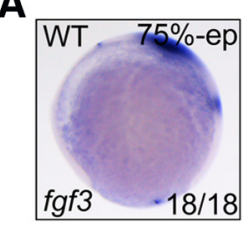

D

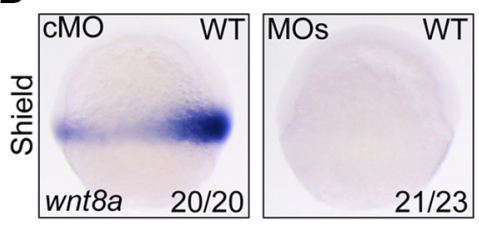

B

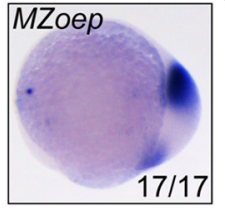

\section{E}

C
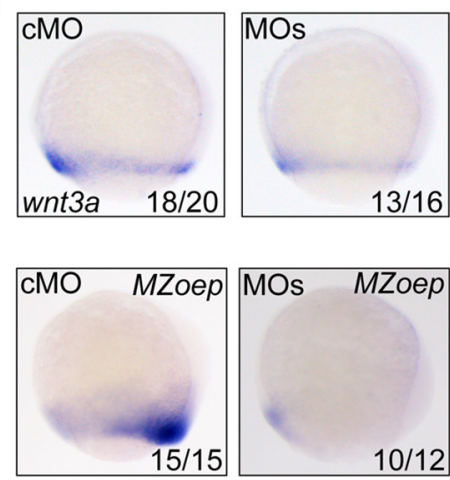

F
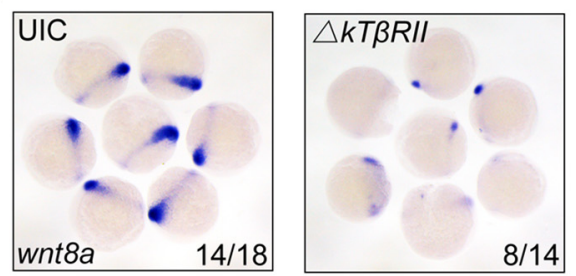

G
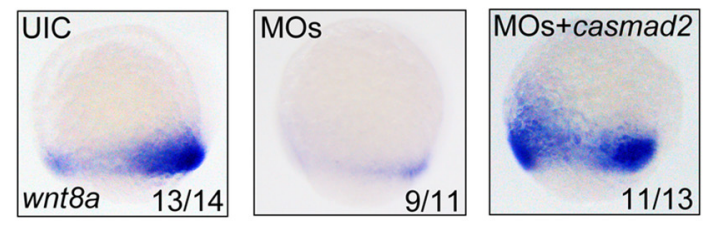

Figure 5. Identification of Bptf and TGF- $\beta / \mathrm{Smad} 2$ coregulated genes during neural posteriorization. $\boldsymbol{A}-\boldsymbol{C}$, The expression pattern of fgf3, fgf8a, and wnt3a at $75 \%$ epiboly stages in wild-type, MZoep mutant, and bptf MOs injected embryos. All embryos were shown in lateral view with dorsal to the right. $\boldsymbol{D}, \boldsymbol{E}$, The expression of wnt8a is regulated by bptf, but not Nodal, signaling. wnt8a expression was assessed at shield $(\boldsymbol{D})$ and $75 \%$ epiboly stages $(\boldsymbol{E})$ by in situ hybridization in wild-type $(\boldsymbol{D}, \boldsymbol{E})$ and $M Z$ Zoep $(\boldsymbol{E})$ embryos injected with cM0 or bptf $\mathrm{M} 0$ s. $\boldsymbol{F}$, Reduction of wnt8a expression in $\triangle k T \beta R / / m R N A$-injected MZoep mutant embryos. A group of embryos at $75 \%$ epiboly stage were shown. G, Ectopic expression of casmad 2 restores bptf M0s-induced decrease of wnt8a expression. MZoep mutant embryos were injected with $8 \mathrm{ng}$ bptf MOs alone or together with $50 \mathrm{pg}$ casmad $2 \mathrm{mRNA}$ at the one-cell stage and harvested at $75 \%$ epiboly stage for in situ hybridization.

scriptional regulation of $w n t 8 a$ by Bptf and $\operatorname{Smad} 2$, the putative 1101-bp wnt8a promoter (1101-P-wnt8a), which possesses a margin-specific enhancer activity sufficient to drive wnt8a expression in ventrolateral mesoderm during neural AP patterning (Narayanan and Lekven, 2012), was cloned into pGL3-Basic luciferase reporter plasmid to make the construct 1101-P-luc (Fig. $7 A$ ). The transcriptional activity of $1101-P$-luc was reduced by nearly 10-fold in $b p t f$ morphants, which suggests that the $1101 \mathrm{bp}$ $w n t 8 a$ promoter responds well to Bptf activity (Fig. $7 B$ ). Next, serial promoter truncations were generated and injected into embryos together with cMO or bptf MOs (Fig. 7A). Results showed that the transcriptional activity of the truncated promoters lacking the distal region of $177 \mathrm{bp}$ was much lower and lost the response to $b p t f$ knockdown (Fig. $7 B$ ). As Bptf can regulate transcription through direct binding to DNA (Jordan-Sciutto et al., 1999; Jones et al., 2000), we searched consensus Bptf binding motifs ( $5^{\prime}$-NNMACAACAMN-3') in that 177 bp region using Genomatix MatInspector (Genomatix database; http://www. genomatix.de), and found a candidate site ( $5^{\prime}$-ACAAAAACACT$3^{\prime}$, positioning from -961 to -951$)$. The AACA $>$ GGAG mutation within this putative Bptf-binding site of 1101-P-luc construct, generating the mutant construct 1101-mb-P-luc (Fig. $7 A$ ), led to a remarkable reduction of reporter expression in wildtype embryos, which was not deteriorated by coinjection of $b p t f$ MOs (Fig. 7C). These results indicate that the identified Bptf binding site is critical for $w n t 8 a$ transcription.

Similar analysis was performed to search Smad2 binding sites in the $w n t 8 a$ promoter. Luciferase reporter assays in embryos revealed that the region of -924 to -657 was required for response to stimulation by casmad2 overexpression (Fig. 7D). Subsequent sequence analysis and reporter assays identified the Smad2/4 binding site ( $5^{\prime}$-CAGAC- $\left.3^{\prime}\right)$ from -921 to -917 as an essential regulatory element (Fig. $7 E$ ).

To verify the in vivo binding of Bptf and Smad2 to the wnt8a promoter, we performed ChIP assays in zebrafish embryos using
Bptf and Smad2 antibodies respectively. As shown in Figure 7F, endogenous Bptf and Smad2 were bound to the -1072 to -892 region of the $w n t 8 a$ promoter, but not to a control region (643 to 842 ), indicating that $w n t 8 a$ is a direct target of Bptf and TGF- $\beta$ / Smad 2 in zebrafish embryos. We further tested whether activated Smad2 and Bptf were mutually required for associating with the $w n t 8 a$ promoter. As shown in Figure $7 G$, inhibition of TGF- $\beta$ signaling by SB435412 treatment or $\Delta k T \beta R I I$ mRNA overexpression did not affect binding of Bptf to the wnt 8 a promoter. bptf knockdown obviously decreased the binding of Bptf to wnt8a promoter (Fig. $7 H$ ) but did not disrupt binding of Smad2 (Fig. $7 I)$. These results suggest that Bptf and Smad2 independently bind to their own binding sites in the wnt8a promoter but can function synergistically to regulate $w n t 8 a$ transcription.

\section{Chromatin remodeling complex NURF is necessary for Bptf and Smad 2 regulation of $w n t 8 a$ expression and neural posteriorization}

NURF is an ATP-dependent chromatin remodeling complex that contains a NURF301 homolog (BPTF in humans), an ATPase subunit of the ISWI family (Smarca1/SNF2L in mammals), and additional subunits. To understand the relationship between Bptf and NURF complex during neural posteriorization, we tried to interfere with the endogenous chromatin remodeling activity of NURF complex in zebrafish embryos by overexpressing smarca1K174R, a dominant-negative zebrafish SNF2L homolog, which contains a point mutation in the nucleotide-binding P-loop motif resulting in loss of the ATPase activity (Corona et al., 1999; Barak et al., 2003). Similar to bptf morphants, embryos injected with smarcalK174R mRNA exhibited a marked reduction of $w n t 8 a$ expression (Fig. $8 A$ ) and inefficient posteriorization of the neuroectoderm (Fig. $8 B-D$ ). These observations revealed that the catalytic subunit of NURF complex is indispensable for $w n t 8 a$ expression and neuroectodermal posteriorization. 
A
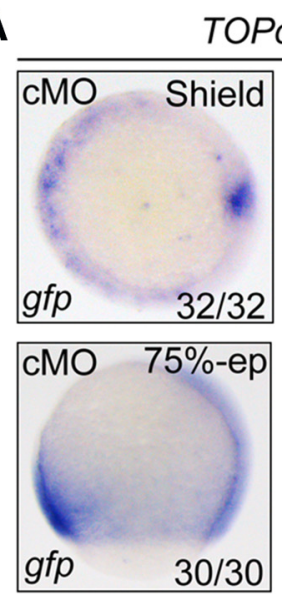

C
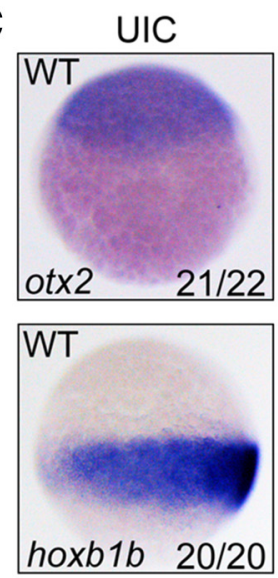

D
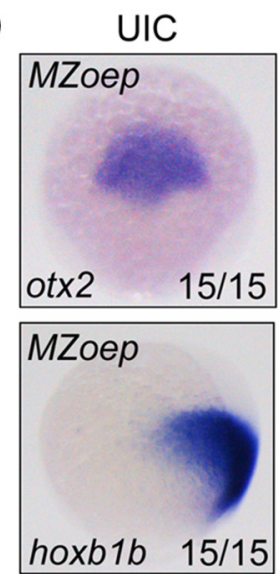
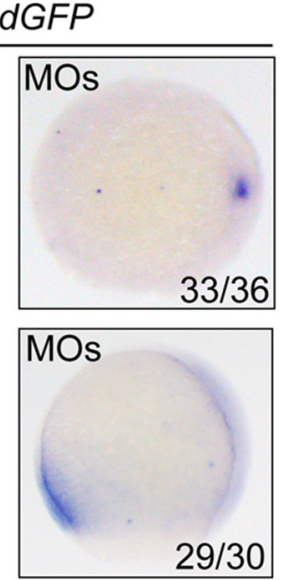

$29 / 30$
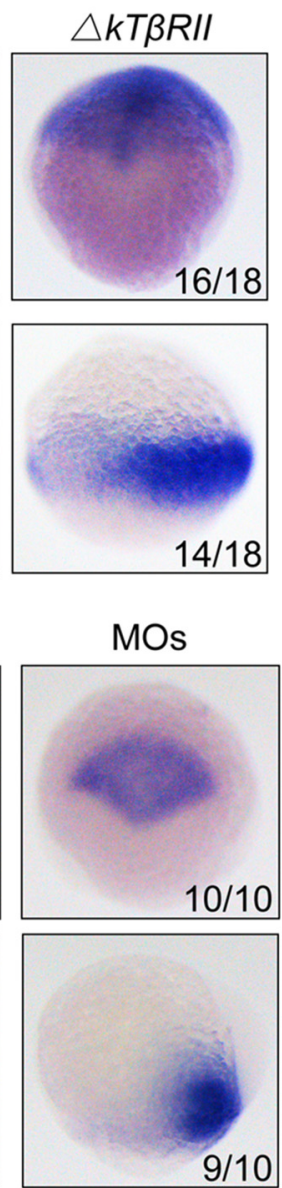

B
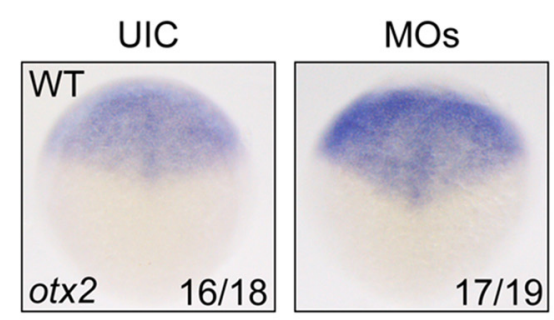

$\mathrm{MOs}+$ wnt8a
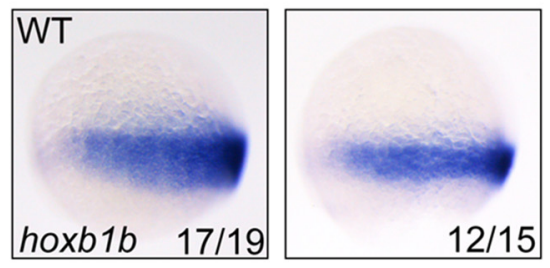

$\mathrm{MOs}+\triangle k T \beta R I I$

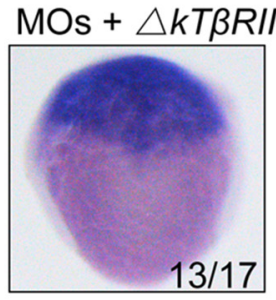

$+w n t 8 a$
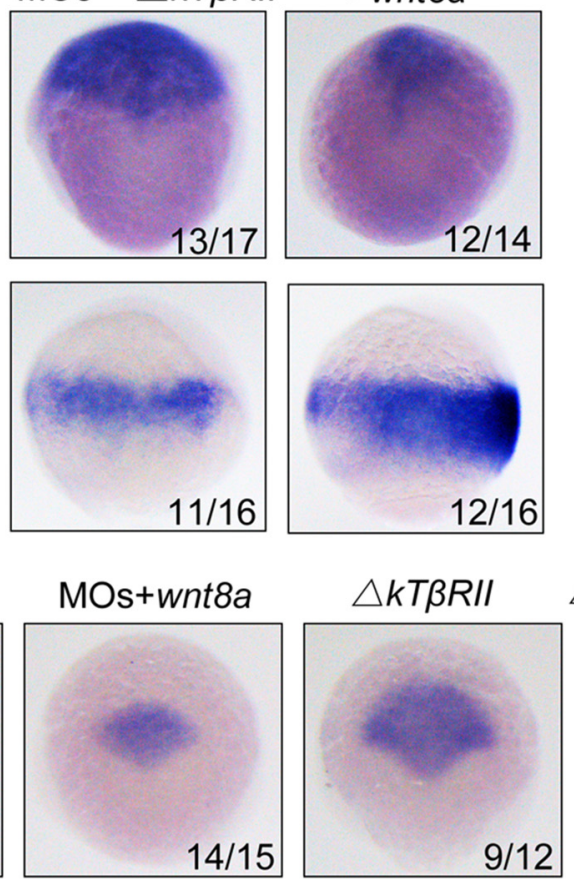

$\triangle k T \beta R I I+w n t 8 a$
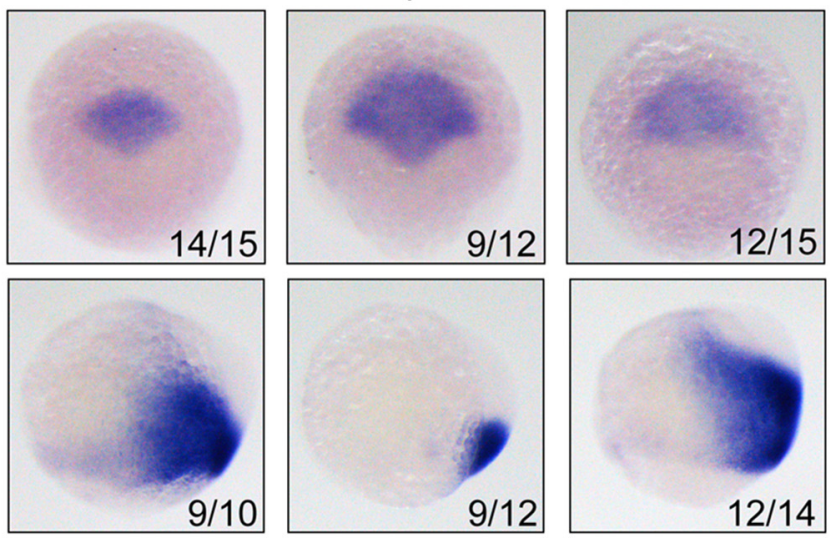

Figure 6. Bptf and TGF- $\beta / \mathrm{Smad} 2$ posteriorize the neuroectoderm via wnt $8 a . A, \mathrm{Wnt} / \beta$-catenin signaling is reduced in bptf morphants. gfp expression was examined at shield and $75 \%$ epiboly stages by in situ hybridization in TOPdGFP transgenic fish embryos injected with $\mathrm{CMO}$ or bptf MOs. Top, Animal-pole views with dorsal to the right. Bottom, Lateral views with dorsal to the right. $\boldsymbol{B}$, Expression of otx2 and hoxb1b at 75\% epiboly stage in wild-type embryos injected with bptf MOs alone or together with $25 \mathrm{pg}$ wnt8a mRNA. Injection of wnt8a mRNA could compromise the expansion of the anterior neuroectoderm and the loss of posterior neuroectoderm in bptf morphants. C, D, Neural patterning defects resulting from inactivation of both $b p t f$ and TGF- $\beta$ signaling were rescued by coinjection of wnt8a mRNA. Wild-type embryos $(\boldsymbol{C})$ or MZoep mutants $(\boldsymbol{D})$ were injected with indicated MOs or RNAs at the one-cell stage and harvested at $75 \%$ epiboly stage for in situ hybridization using otx2 and hoxb16 probes. Injection doses were as follows: bptf MOs, $8 \mathrm{ng} ; \triangle k T \beta R / l \mathrm{mRNA}, 400 \mathrm{pg}$; wnt8a mRNA, $25 \mathrm{pg}$.

NURF is known to be directly recruited by site-specific transcription factors (Xiao et al., 2001; Badenhorst et al., 2005). We hypothesized that the interaction of Bptf with Smad2 can enhance the recruitment of other NURF components, including Smarca1. To verify this hypothesis, coimmunoprecipitation assays were performed to assess the impact of Smad2 on the recruitment of Smarca1 to Bptf in HEK293 cells. There was a basal level interaction between Bptf and Smarca1, which was considerably enhanced in the presence of Smad2 (Fig. 8E). We then analyzed the functional importance of interactions among Bptf, Smad2, and Smarcal in zebrafish embryos. Coinjection of wild-type smarcal mRNA restored both $w n t 8 a$ and hoxblb expression in bptf morphants (Fig. 8F). Meanwhile, overexpression of smarca 1K174R partially prevented the Smad2-mediated recovery of wnt $8 a$ and 


\section{A}
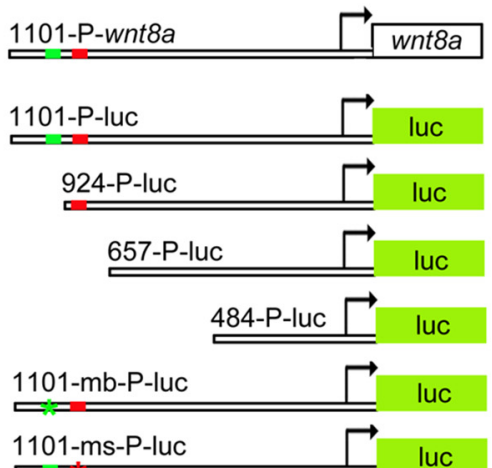

Bptf $\square$ Smad2/4

* mutant Bptf $\quad$ * mutant Smad2/4

D

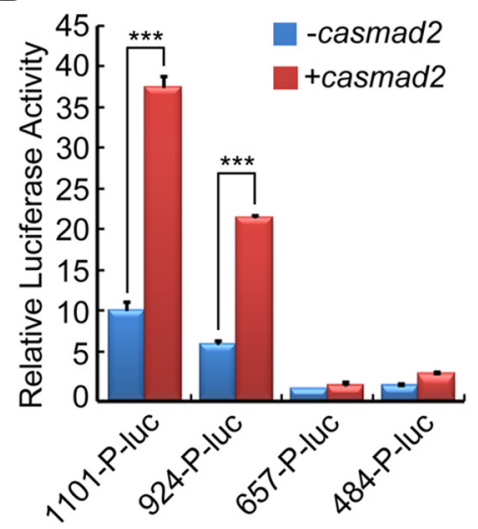

G

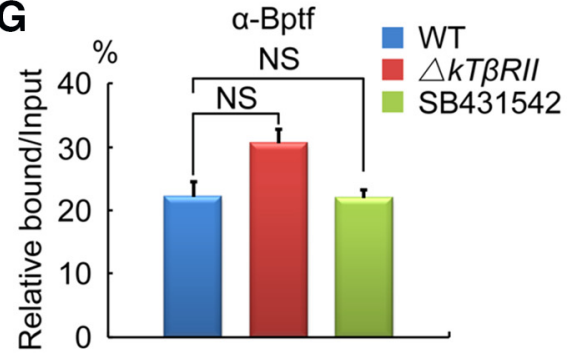

B

E
$\mathrm{cMO}$

MOs
C

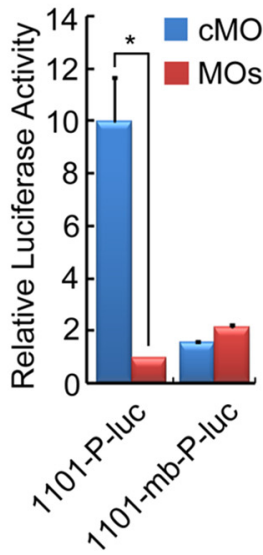

$\mathbf{F}$

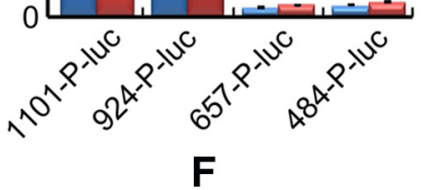

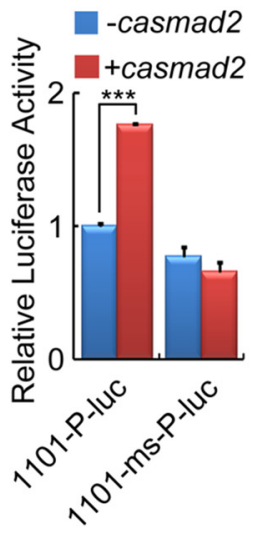

H

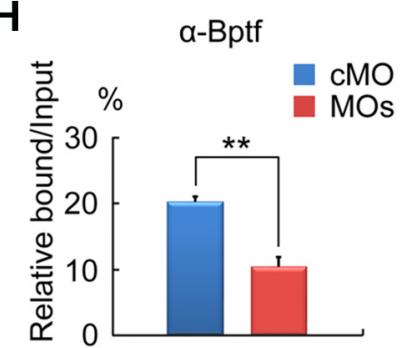

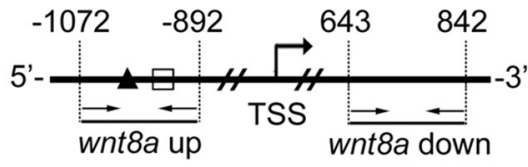

A Bptf $\square$ Smad2/4

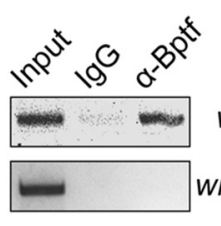

wnt8a up

wnt8a down

I

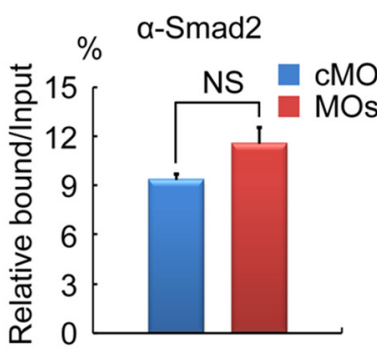

Figure 7. Bptf and Smad2 regulate wnt8a expression through distinct cis-elements. $A$, Schematic illustration of wnt8a promoter-driven luciferase reporters. $B, C$, The Bptf-binding element is essential for wnt8a transcription. Wild-type embryos were injected with indicated MOs and wnt8a promoter-driven reporter constructs at one-cell stage and then harvested and lysed at $75 \%$ epiboly stage for luciferase activity analysis. The -1101 to -924 promoter region is important for wnt8a transcription (B). 1101-mb-P-luc showed a much lower luciferase activity than the corresponding wild-type reporter (C).D, The -924 to -657 region of the wnt8a promoter is essential for responding to casmad 2 overexpression. The indicated constructs and casmad $2 \mathrm{mRNA}$ were injected into embryos at one-cell stage, and the relative luciferase activity was measured at 75\% epiboly stage. $E$, The Smad2/4-binding site is essential for responding to casmad2 overexpression. $1101-m s-P$-luc or the related wild-type reporter 1101-P-luc was injected into embryos, and their responsiveness to overexpressed casmad2 was measured. Data represent the mean \pm SD for triplicate experiments. ${ }^{*} p<0.05$ (Student's $t$ test). ${ }^{* *} p<0.01$ (Student's $t$ test). ${ }^{* * *} p<0.001$ (Student's $t$ test). $F$, Bptf and Smad2 specifically bind to the wnt8a promoter. ChIP assays were performed with control IgG and antibodies against Bptf or Smad2/3 in wild-type embryos. The immunoprecipitated DNA was amplified by semiqPCR with the primers recognizing specific regions denoted in the top. wnt8a up, a region upstream of the wnt8a TSS, which contains Bptf and Smad2/4 binding motifs; wnt8a down, a region downstream of the wnt8a TSS. G, ChIP experiments were performed in wild-type, $\triangle k T \beta R / / m R N A$-injected, or SB431542-treated embryos using anti-Bptf antibody. The immunoprecipitated DNA was amplified by qPCR with primers to detect the region upstream of the wnt8a TSS. NS, Nonsignificant. $\boldsymbol{H}, \boldsymbol{I}$, Wild-type embryos were injected with $\mathrm{CM} 0$ or bptf MOs at the one-cell stage and harvested at $75 \%$ epiboly stage for $C \mathrm{Ch}$ IP experiments with anti-Bptf $(\boldsymbol{H})$ or anti-Smad2/3 (I) antibodies. The immunoprecipitated DNA was subjected to qPCR with primers indicated in $\mathbf{G}$.

hoxb1b expression in bptf morphants (Fig. $8 F$ ). Together, these results indicate that interaction of Bptf with Smad2 facilitates the recruitment of NURF complex to regulate $w n t 8 a$ expression and neural posteriorization.

\section{Inhibited bptf expression or TGF- $\beta$ signaling increases nucleosome density in the $w n t 8 a$ promoter}

Our data thus far demonstrated the physical association and functional link between Bptf-containing NURF complex and Smad 2 on $w n t 8 a$ expression during neural posteriorization. To analyze nucleosome positioning pattern in the wnt8a promoter, we performed MNase mapping assay as previously described ( $\mathrm{Li}$ et al., 2010, 2013). In this assay, chromatin isolated from pooled embryos was digested into mononucleosomes with MNase (Fig. $9 A$ ), and resulted DNA fragments were extracted and amplified using 30 separate overlapping primer pairs, spanning $\sim 1000 \mathrm{bp}$ DNA stretch around the identified Bptf and Smad2 binding sites in the wnt8a promoter. We observed five nucleosome binding sites, named N1, N2, N3, N4, and N5, respectively, in the examined region in wild-type embryos. Interestingly, either of the Bptf 
A

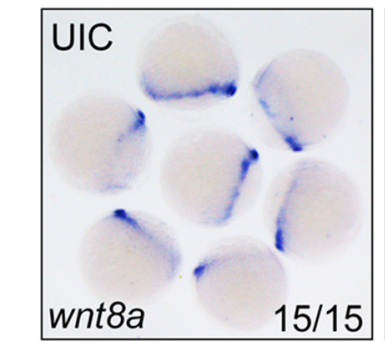

C

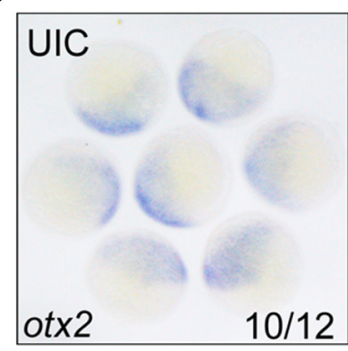

D

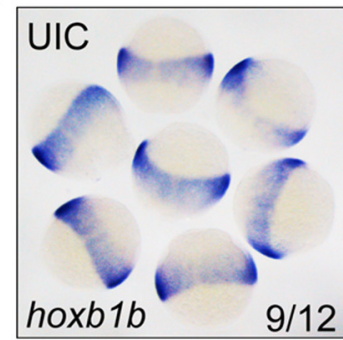

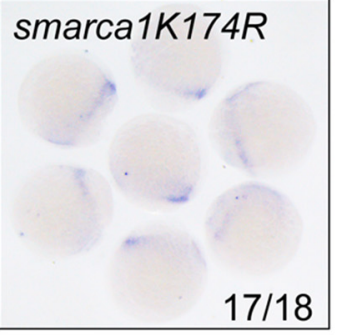

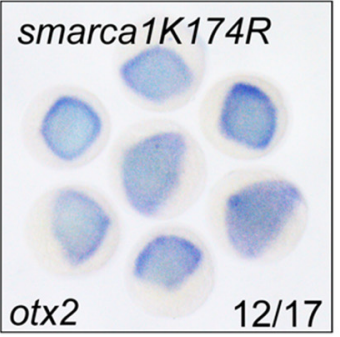

otx

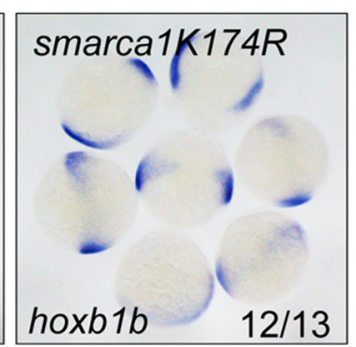

B
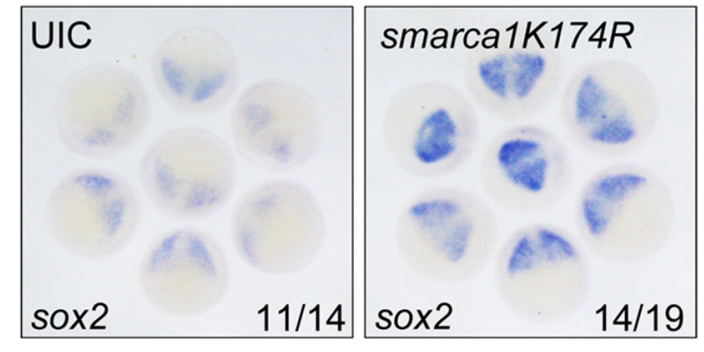

E

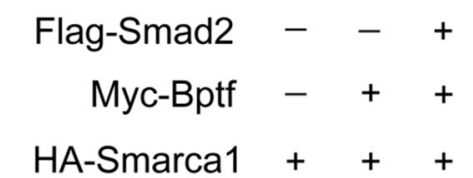

IP: $\alpha-M y c$

WB: $\alpha-H A$

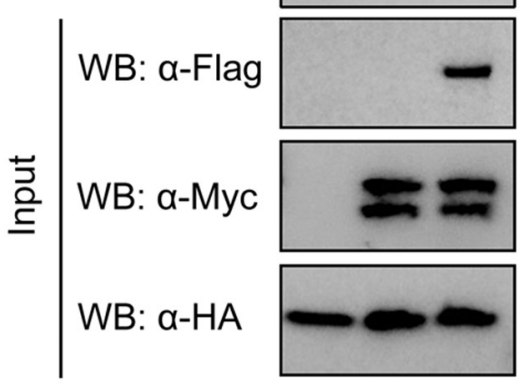

MOs+casmad2

F

UIC
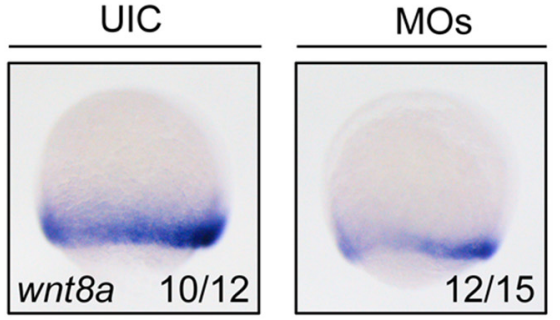

MOs+smarca1

MOs+casmad2

+smarca1K174R
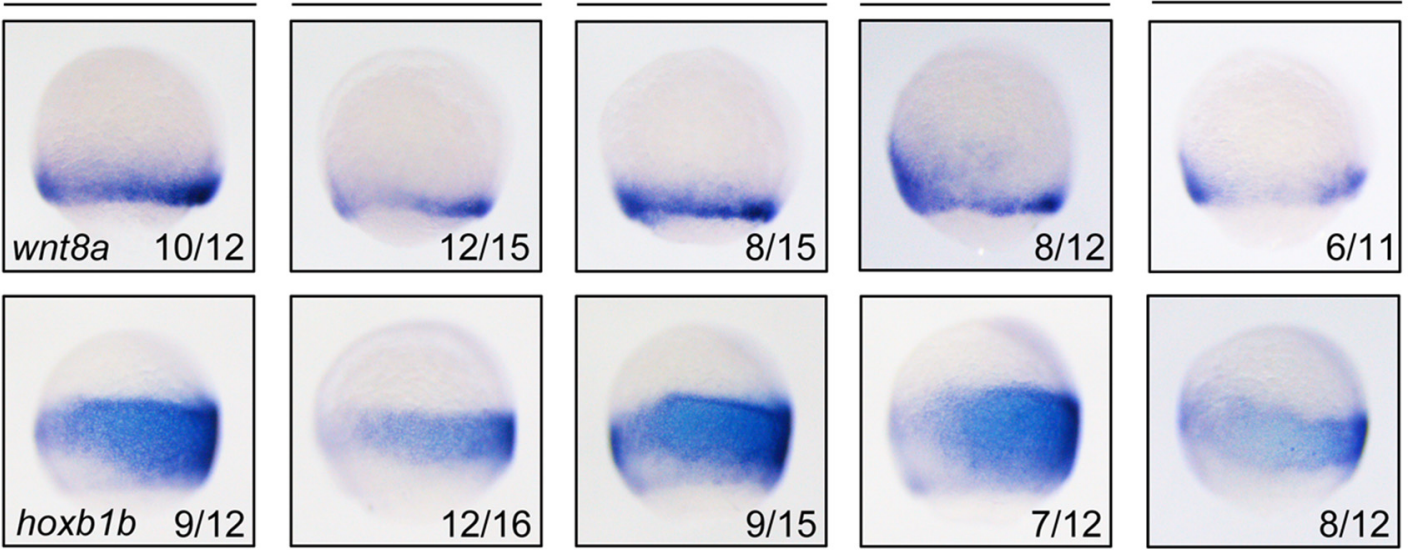

$6 / 11$
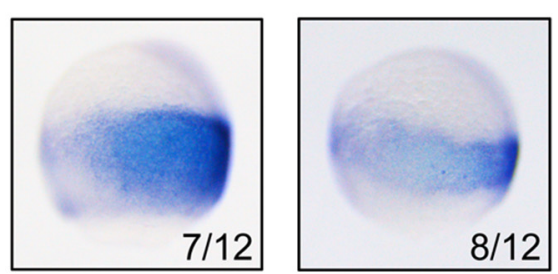

Figure 8. Smarca1 is required for wnt8a expression and neural posteriorization. $A$, Interference in smarca 1 inhibits wnt8a expression. Uninjected and smarca1K174R mRNA-injected (125 pg) embryos were probed at $75 \%$ epiboly stage for wnt8a expression by in situ hybridization. $\boldsymbol{B}-\boldsymbol{D}$, smarca 1 K174R overexpression disrupts neuroectodermal AP patterning. One-cell stage embryos were injected with $125 \mathrm{pg}$ smarca $1 \mathrm{~K} 174 \mathrm{R} \mathrm{mRNA}$ and harvested at $75 \%$ epiboly stage for in situ hybridization to detect the anterior neural markers sox2 $(\boldsymbol{B})$ and otx2 (C) and the posterior neural marker hoxb1b (D).E, Smad2 enhances the association of Bptf and Smarca1. HEK293T cells were transfected with combinations of plasmids expressing Myc-Bptf, HA-Smarca1, and Flag-Smad2 as indicated. After $48 \mathrm{~h}$, cells were lysed for immunoprecipitation with anti-Myc antibody. F, Smarca1 functions on Bptf- and Smad2-mediated wnt8a expression and neural posteriorization. Embryos were injected with indicated MOs and mRNAs at the one-cell stage and harvested at 75\% epiboly stage for in situ hybridization to detect alteration of wnt8a and hoxb1b expression. Embryos were orientated laterally with dorsal to the right. Injection doses are as follows: bptf M0s, $8 \mathrm{ng}$; smarca1 mRNA, 250 pg; smarca1K174R mRNA, 125 pg; casmad2 mRNA, 50 pg.

and Smad2 binding sites was not located in the nucleosome-free areas but instead fallen into the N3 site (Fig. 9B). We speculate that nucleosome packaging may be relaxed only in lateral/paraxial mesodermal precursors within which wnt8a is expressed. Indeed, a slight but steady increase in DNA amount was detected at $\mathrm{N} 2$ and N3 sites in bptf morphants, indicating a nucleosome repositioning event occurred in the wnt8a promoter (Fig. 9B). We further assayed histone occupancy on the wnt8a promoter through ChIP assays with antibody against histone $\mathrm{H} 3$ and found that the occupancy of histone $\mathrm{H} 3$ was enhanced in the region containing Bptf- and Smad2-binding sites in bptf morphants (Fig. $9 B^{\prime}$ ). Consistent with the pivotal role of TGF- $\beta / S \operatorname{mad} 2$ in the recruitment of Bptf-containing NURF complex, interference with TGF- $\beta$ signaling by overexpression of $\Delta k T \beta R I I$ in embryos 
A

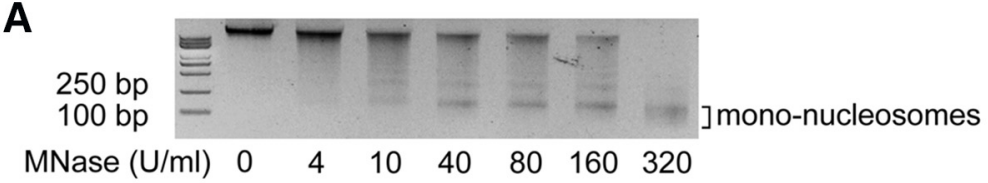

B
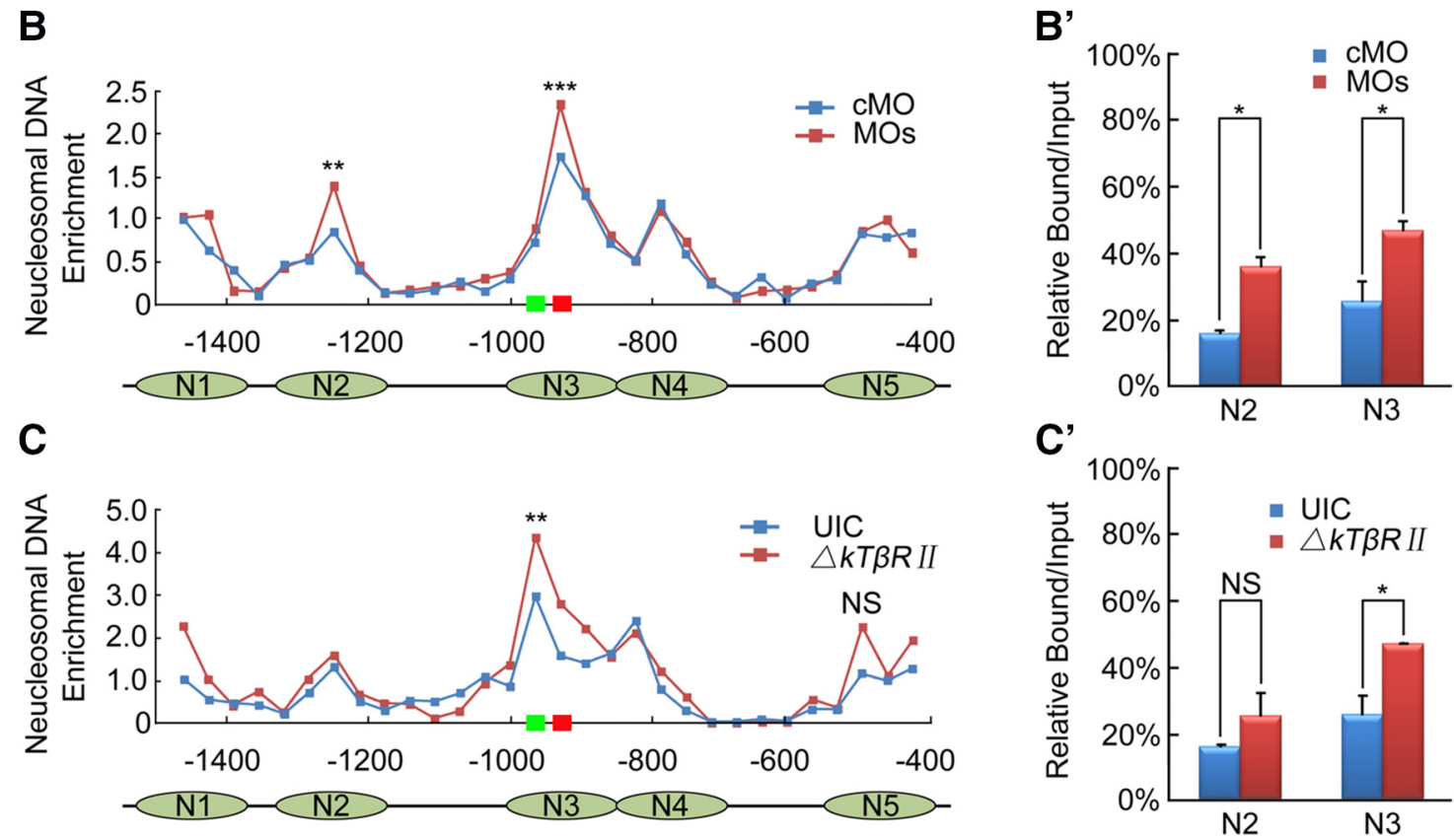

Figure 9. Inactivation of $b$ tf or TGF- $\beta$ signaling induces nucleosome repositioning within the wnt8a promoter. A, MNase digestion of chromatin isolated from embryos at $75 \%$ epiboly stage. Digestion with 320 units per milliliters of MNase for 30 min was appropriate to produce mononucleosome-sized DNAs. $B, C$, The dynamic changes of nucleosomal positions at the wnt8a promoter in bptf morphants $(\boldsymbol{B})$ or $\Delta$ kT $\beta R l$-overexpressing embryos (C). There were five positioned nucleosomes (N1, N2, N3, N4, and N5) within the -1449 to -416 region of the wnt8a promoter in CM0-injected embryos. Bptf (green) and Smad2 (red) binding motifs were located in the DNA sequences occupied by N3. A solid increase in DNA amount was detected at N3 positioning site in bptf morphants and $\Delta k T \beta R I I$-overexpressing embryos. NS, Nonsignificant. ${ }^{* *} p<0.01$ (Student's $t$ test). ${ }^{* * *} p<0.001$ (Student's $t$ test). $\boldsymbol{B}^{\prime}, \boldsymbol{C}^{\prime}$, Injection of bptf MOs or $\Delta k T \beta R I / \mathrm{mRNA}$ results in an increase in histone density over the positioned N3 within the wnt8a promoter. Embryos were injected with $8 \mathrm{ng}$ bptf MOs or $400 \mathrm{pg} \Delta \mathrm{kT} \beta R / / \mathrm{mRNA}$ at one-cell stage and harvested at $75 \%$ epiboly stage. The resultant chromatin was extracted and subjected to ChIP assays with anti-H3 antibody followed by qPCR. NS, Nonsignificant. ${ }^{*} p<0.05$ (Student's $t$ test).

caused a solid increase of histone density at the N3 site (Fig. $\left.9 C, C^{\prime}\right)$. Interestingly, increased nucleosome occupancy was also observed at the N2 site in bptf morphants but not in $\Delta k T \beta R I I$ mRNA-injected embryos (Fig. $9 B, C^{\prime}$ ), indicating a subtle difference between Bptf and TGF- $\beta /$ Smad2 signaling in regulating $w n t 8 a$ transcription. Together, these data suggest that both Bptf and TGF- $\beta /$ Smad 2 are required for nucleosome remodeling at $w n t 8 a$ promoter during neural patterning.

\section{Discussion}

The neuroectoderm patterning along the body axis is an important step of the CNS development. So far, little has been known about implications of epigenetic factors in this neural patterning process. In the present study, we find that zebrafish bptf has a crucial function in transforming the anterior neuroectoderm fate to the posterior neuroectoderm fate. The effect of $b p t f$ on posteriorization of the neuroectoderm is closely linked to the chromatin remodeling activity of NURF complex. This is supported by the finding that inhibition of endogenous chromatin remodeling activities of NURF complex by injection of dominant-negative smarca $1 \mathrm{mRNA}$ results in neural patterning defects similar to $b p t f$ knockdown. Consistently, coinjection of wild-type smarcal mRNA well rescued the neural posteriorization defects caused by $b p t f$ knockdown. More importantly, loss of $b p t f$ function could enhance the nucleosome density in the wnt8a promoter and subsequently restrain transcription. Lan et al. (2007) previously reported that inactivation of the histone demethylase UTX and
JMJD led to an imbalance of H3K27 methylation, mis-regulation of hox genes, and a striking posterior developmental defect, which provides an indirect evidence for involvement of H3K27 methylation in neural patterning.

Smad2 and Smad3 are intracellular effectors of TGF- $\beta$, Activin, and Nodal signals (Massagué et al., 2005; Wu and Hill, 2009). One previous report shows that Bptf, the largest subunit of NURF complex, directly interacts with and activates target gene transcription of Smad2/3 (Landry et al., 2008). Our previous work indicates that the consensus binding motif of Bptf exists most frequently in Smad2-bound promoter regions (Liu et al., 2011). In agreement with these previous findings, we reveal that zebrafish Bptf physically and functionally interacts with Smad2 during neural AP patterning. The contribution of Bptf to the Smad2-regulated neuroectodermal posteriorization is supported by the findings that the interaction of Bptf with Smad2 facilitates the recruitment of Smarca1/SNF2L, the ATPase subunit of NURF complex, and that overexpression of dominant-negative smarcal inhibits the Smad2-mediated recovery of neural patterning defects in $b p t f$ morphants.

Zebrafish wnt8a is required during gastrulation to generate the AP pattern of the nascent neuroectoderm (Erter et al., 2001; Lekven et al., 2001). There is a margin-specific enhancer located to the -989 to -497 region upstream of the wnt8a TSS. The expression of wnt $8 a$ can be divided into two phases: Phase I, from $30 \%$ epiboly to $60 \%$ epiboly (4.7-6.5 hpf), depending on Nodal 
activation through the margin enhancer; and Phase II, from 70\% epiboly until the end of gastrulation (7-10 hpf), requiring $n t l$ activation of the margin enhancer independently of Nodal signaling (Narayanan and Lekven, 2012). Transplantation experiments indicate that the presumptive hindbrain precursors acquire a regional identity between shield stage and mid-gastrulation stages (6-8 hpf) (Woo and Fraser, 1998; Appel, 2000). We find that $w n t 8 a$ is normally expressed in MZoep mutant embryos at $75 \%$ epiboly stage, which support the idea that the wnt8a expression during neural patterning is not regulated by Nodal signal. The inhibition of $w n t 8 a$ expression by specific interference with $b p t f$ activity or TGF- $\beta$ signal transduction in wild-type and MZoep mutant embryos indicates that $w n t 8 a$ is a downstream target gene of $b p t f$ and TGF- $\beta /$ Smad 2 . In contrast to $w n t 8 a$, the ventrolateral mesodermal expression of $f g f 8$ and $w n t 3 a$ remains almost unchanged in $b p t f$ morphants, suggesting the specificity of $b p t f$ in regulating $w n t 8 a$ expression.

To our surprise, the expression of $w n t 8 a$ is also significantly decreased in $b p t f$ morphants at shield stage. As the expression of wnt8a depending on Nodal activation at this stage (Narayanan and Lekven, 2012) and Bptf is necessary for Nodal/Smadmediated mesendoderm differentiation of mouse embryonic stem cells (Landry et al., 2008), we speculate that the expression of $w n t 8 a$ at shield stage is regulated by Bptf and Nodal/Smad but not the TGF- $\beta /$ Smad pathway. The ventrolaterally expressed $w n t 8 a$ functions in restricting the size of the dorsal organizer (Lekven et al., 2001; Ramel and Lekven, 2004), but the decreased expression of $w n t 8 a$ in $b p t f$ morphants at early stage did not result in visible dorsoventral patterning defects. We think that loss of bptf may inhibit the activity of Nodal/Smad signaling or the expression of some unknown $b p t f$ target genes, which is essential in dorsoventral axis determination to counteract the dorsalization effects induced by the decreased expression of wnt8a.

With luciferase reporter assays and ChIP-PCR experiments, we demonstrate that Bptf and Smad2 bind to their respective consensus binding motifs in the wnt8a promoter and elevate $w n t 8 a$ transcription. Interestingly, the identified Bptf and Smad2 binding motifs lie in the margin-specific enhancer, which is essential for the Phase II expression of wnt8a (Narayanan and Lekven, 2012). Unlike another bromodomain and plant homeodomain finger-containing protein TRIM33, which is essential for Smad2/3 binding to AREs in poised promoters during stem cell differentiation (Xi et al., 2011), Bptf and Smad2 independently bind to the wnt 8 a promoter. The adjacent location of their binding motifs may provide an appropriate space for their interaction and cooperative function. In addition to interact with Bptf, Smad2 also physically associates with Smarca1/SNF2L as previously reported (Landry et al., 2008; Xi et al., 2008). Our coimmunoprecipitation results indicate that the interaction of Bptf with Smad2 could enhance the recruitment of Smarcal/ SNF2L to build up the functional NURF complex. Depletion of $b p t f$ expression or blocking TGF- $\beta$ signal transduction causes an increase of the nucleosome density around Bptf and Smad 2 binding motifs within the wnt8a promoter, suggesting a role in chromatin remodeling. Therefore, we propose that Bptf functions as a coactivator of Smad2 to recruit other components of chromatin remodeling complex NURF, resembling the histone acetyltransferase p300, which is required for Smad2-mediated transcription to acetylate nucleosomal histones on TGF- $\beta$ responsive promoters (Ross et al., 2006).

In conclusion, our study discovers that zebrafish bptf and TGF- $\beta /$ Smad 2 signaling cooperatively promote neural posteriorization. TGF- $\beta$-activated Smad 2 binds to $w n t 8 a$ promoter and facilitates the recruitment of Bptf-containing NURF complex to drive $w n t 8 a$ expression and neural patterning. The model proposed here provides a mechanism whereby $b p t f$ and TGF- $\beta /$ Smad 2 coregulate neural patterning in zebrafish embryos. In the future, it will be interesting to investigate whether this model applies to the neuroectoderm patterning in other animals.

\section{References}

Appel B (2000) Zebrafish neural induction and patterning. Dev Dyn 219: 155-168. CrossRef Medline

Badenhorst P, Xiao H, Cherbas L, Kwon SY, Voas M, Rebay I, Cherbas P, Wu C (2005) The Drosophila nucleosome remodeling factor NURF is required for Ecdysteroid signaling and metamorphosis. Genes Dev 19: 2540-2545. CrossRef Medline

Barak O, Lazzaro MA, Lane WS, Speicher DW, Picketts DJ, Shiekhattar R (2003) Isolation of human NURF: a regulator of Engrailed gene expression. EMBO J 22:6089-6100. CrossRef Medline

Brand T, MacLellan WR, Schneider MD (1993) A dominant-negative receptor for type beta transforming growth factors created by deletion of the kinase domain. J Biol Chem 268:11500-11503. Medline

Buckles GR, Thorpe CJ, Ramel MC, Lekven AC (2004) Combinatorial Wnt control of zebrafish midbrain-hindbrain boundary formation. Mech Dev 121:437-447. CrossRef Medline

Cannon JE, Place ES, Eve AM, Bradshaw CR, Sesay A, Morrell NW, Smith JC (2013) Global analysis of the haematopoietic and endothelial transcriptome during zebrafish development. Mech Dev 130:122-131. CrossRef Medline

Cheah FS, Jabs EW, Chong SS (2005) Genomic, cDNA, and embryonic expression analysis of zebrafish transforming growth factor beta 3 (tgfbeta3). Dev Dyn 232:1021-1030. CrossRef Medline

Chen RH, Ebner R, Derynck R (1993) Inactivation of the type II receptor reveals two receptor pathways for the diverse TGF-beta activities. Science 260:1335-1338. CrossRef Medline

Corona DF, Längst G, Clapier CR, Bonte EJ, Ferrari S, Tamkun JW, Becker PB (1999) ISWI is an ATP-dependent nucleosome remodeling factor. Mol Cell 3:239-245. CrossRef Medline

Cox WG, Hemmati-Brivanlou A (1995) Caudalization of neural fate by tissue recombination and bFGF. Development 121:4349-4358. Medline

Dal-Pra S, Fürthauer M, Van-Celst J, Thisse B, Thisse C (2006) Noggin1 and Follistatin-like2 function redundantly to Chordin to antagonize BMP activity. Dev Biol 298:514-526. CrossRef Medline

Dodd A, Chambers SP, Love DR (2004) Short interfering RNA-mediated gene targeting in the zebrafish. FEBS Lett 561:89-93. CrossRef Medline

Dorsky RI, Sheldahl LC, Moon RT (2002) A transgenic Lefl/beta-catenindependent reporter is expressed in spatially restricted domains throughout zebrafish development. Dev Biol 241:229-237. CrossRef Medline

Erter CE, Wilm TP, Basler N, Wright CV, Solnica-Krezel L (2001) Wnt8 is required in lateral mesendodermal precursors for neural posteriorization in vivo. Development 128:3571-3583. Medline

Escher D, Bodmer-Glavas M, Barberis A, Schaffner W (2000) Conservation of glutamine-rich transactivation function between yeast and humans. Mol Cell Biol 20:2774-2782. CrossRef Medline

Feldman B, Gates MA, Egan ES, Dougan ST, Rennebeck G, Sirotkin HI, Schier AF, Talbot WS (1998) Zebrafish organizer development and germ-layer formation require nodal-related signals. Nature 395:181-185. CrossRef Medline

Goller T, Vauti F, Ramasamy S, Arnold HH (2008) Transcriptional regulator $\mathrm{BPTF} / \mathrm{FAC1}$ is essential for trophoblast differentiation during early mouse development. Mol Cell Biol 28:6819-6827. CrossRef Medline

Gritsman K, Zhang J, Cheng S, Heckscher E, Talbot WS, Schier AF (1999) The EGF-CFC protein one-eyed pinhead is essential for nodal signaling. Cell 97:121-132. CrossRef Medline

Hagos EG, Fan X, Dougan ST (2007) The role of maternal Activin-like signals in zebrafish embryos. Dev Biol 309:245-258. CrossRef Medline

Hammerschmidt M, Pelegri F, Mullins MC, Kane DA, van Eeden FJ, Granato M, Brand M, Furutani-Seiki M, Haffter P, Heisenberg CP, Jiang YJ, Kelsh RN, Odenthal J, Warga RM, Nüsslein-Volhard C (1996) dino and mercedes, two genes regulating dorsal development in the zebrafish embryo. Development 123:95-102. Medline

Hemmati-Brivanlou A, Kelly OG, Melton DA (1994) Follistatin, an antagonist of activin, is expressed in the Spemann organizer and displays direct neuralizing activity. Cell 77:283-295. CrossRef Medline 
Herskowitz I (1987) Functional inactivation of genes by dominant negative mutations. Nature 329:219-222. CrossRef Medline

Inman GJ, Nicolás FJ, Callahan JF, Harling JD, Gaster LM, Reith AD, Laping NJ, Hill CS (2002) SB-431542 is a potent and specific inhibitor of transforming growth factor-beta superfamily type I activin receptor-like kinase (ALK) receptors ALK4, ALK5, and ALK7. Mol Pharmacol 62:65-74. CrossRef Medline

Jia S, Ren Z, Li X, Zheng Y, Meng A (2008) smad2 and smad3 are required for mesendoderm induction by transforming growth factor-beta/nodal signals in zebrafish. J Biol Chem 283:2418-2426. CrossRef Medline

Jia S, Wu D, Xing C, Meng A (2009) Smad2/3 activities are required for induction and patterning of the neuroectoderm in zebrafish. Dev Biol 333:273-284. CrossRef Medline

Jones MH, Hamana N, Shimane M (2000) Identification and characterization of BPTF, a novel bromodomain transcription factor. Genomics 63: 35-39. CrossRef Medline

Jordan-Sciutto KL, Dragich JM, Rhodes JL, Bowser R (1999) Fetal Alz-50 clone 1, a novel zinc finger protein, binds a specific DNA sequence and acts as a transcriptional regulator. J Biol Chem 274:35262-35268. CrossRef Medline

Kelly GM, Greenstein P, Erezyilmaz DF, Moon RT (1995) Zebrafish wnt8 and wnt8b share a common activity but are involved in distinct developmental pathways. Development 121:1787-1799. Medline

Kimmel CB, Ballard WW, Kimmel SR, Ullmann B, Schilling TF (1995) Stages of embryonic development of the zebrafish. Dev Dyn 203:253-310. CrossRef Medline

Kohli G, Hu S, Clelland E, Di Muccio T, Rothenstein J, Peng C (2003) Cloning of transforming growth factor-beta 1 (TGF-beta 1) and its type II receptor from zebrafish ovary and role of TGF-beta 1 in oocyte maturation. Endocrinology 144:1931-1941. CrossRef Medline

Koshida S, Shinya M, Nikaido M, Ueno N, Schulte-Merker S, Kuroiwa A, Takeda H (2002) Inhibition of BMP activity by the FGF signal promotes posterior neural development in zebrafish. Dev Biol 244:9-20. CrossRef Medline

Lamb TM, Knecht AK, Smith WC, Stachel SE, Economides AN, Stahl N, Yancopolous GD, Harland RM (1993) Neural induction by the secreted polypeptide noggin. Science 262:713-718. CrossRef Medline

Lan F, Bayliss PE, Rinn JL, Whetstine JR, Wang JK, Chen S, Iwase S, Alpatov R, Issaeva I, Canaani E, Roberts TM, Chang HY, Shi Y (2007) A histone H3 lysine 27 demethylase regulates animal posterior development. Nature 449:689-694. CrossRef Medline

Landry J, Sharov AA, Piao Y, Sharova LV, Xiao H, Southon E, Matta J, Tessarollo L, Zhang YE, Ko MS, Kuehn MR, Yamaguchi TP, Wu C (2008) Essential role of chromatin remodeling protein Bptf in early mouse embryos and embryonic stem cells. PLoS Genet 4:e1000241. CrossRef Medline

Lekven AC, Thorpe CJ, Waxman JS, Moon RT (2001) Zebrafish wnt8 encodes two wnt8 proteins on a bicistronic transcript and is required for mesoderm and neurectoderm patterning. Dev Cell 1:103-114. CrossRef Medline

Li G, Margueron R, Hu G, Stokes D, Wang YH, Reinberg D (2010) Highly compacted chromatin formed in vitro reflects the dynamics of transcription activation in vivo. Mol Cell 38:41-53. CrossRef Medline

Li Q, Zhang Y, Fu J, Han L, Xue L, Lv C, Wang P, Li G, Tong T (2013) FOXA1 mediates p16(INK4a) activation during cellular senescence. EMBO J 32:858-873. CrossRef Medline

Liu WY, Wang Y, Sun YH, Wang Y, Wang YP, Chen SP, Zhu ZY (2005) Efficient RNA interference in zebrafish embryos using siRNA synthesized with SP6 RNA polymerase. Dev Growth Differ 47:323-331. CrossRef Medline

Liu Z, Lin X, Cai Z, Zhang Z, Han C, Jia S, Meng A, Wang Q (2011) Global identification of SMAD2 target genes reveals a role for multiple coregulatory factors in zebrafish early gastrulas. J Biol Chem 286:2852028532. CrossRef Medline

Loosli F, Staub W, Finger-Baier KC, Ober EA, Verkade H, Wittbrodt J, Baier $\mathrm{H}$ (2003) Loss of eyes in zebrafish caused by mutation of chokh/rx3. EMBO Rep 4:894-899. CrossRef Medline

Maden M (2006) Retinoids and spinal cord development. J Neurobiol 66: 726-738. CrossRef Medline

Massagué J, Seoane J, Wotton D (2005) Smad transcription factors. Genes Dev 19:2783-2810. CrossRef Medline

Mizuguchi G, Tsukiyama T, Wisniewski J, Wu C (1997) Role of nucleosome remodeling factor NURF in transcriptional activation of chromatin. Mol Cell 1:141-150. CrossRef Medline

Narayanan A, Lekven AC (2012) Biphasic wnt8a expression is achieved through interactions of multiple regulatory inputs. Dev Dyn 241:10621075. CrossRef Medline

Niehrs C (1999) Head in the WNT: the molecular nature of Spemann's head organizer. Trends Genet 15:314-319. CrossRef Medline

Nomura M, Li E (1998) Smad2 role in mesoderm formation, left-right patterning and craniofacial development. Nature 393:786-790. CrossRef Medline

Ramel MC, Lekven AC (2004) Repression of the vertebrate organizer by Wnt8 is mediated by Vent and Vox. Development 131:3991-4000. CrossRef Medline

Rentzsch F, Bakkers J, Kramer C, Hammerschmidt M (2004) Fgf signaling induces posterior neuroectoderm independently of Bmp signaling inhibition. Dev Dyn 231:750-757. CrossRef Medline

Ross S, Cheung E, Petrakis TG, Howell M, Kraus WL, Hill CS (2006) Smads orchestrate specific histone modifications and chromatin remodeling to activate transcription. EMBO J 25:4490-4502. CrossRef Medline

Ruthenburg AJ, Li H, Milne TA, Dewell S, McGinty RK, Yuen M, Ueberheide B, Dou Y, Muir TW, Patel DJ, Allis CD (2011) Recognition of a mononucleosomal histone modification pattern by BPTF via multivalent interactions. Cell 145:692-706. CrossRef Medline

Sasai Y, De Robertis EM (1997) Ectodermal patterning in vertebrate embryos. Dev Biol 182:5-20. CrossRef Medline

Sasai Y, Lu B, Steinbeisser H, De Robertis EM (1995) Regulation of neural induction by the Chd and Bmp-4 antagonistic patterning signals in Xenopus. Nature 376:333-336. CrossRef Medline

Schulte-Merker S, Lee KJ, McMahon AP, Hammerschmidt M (1997) The zebrafish organizer requires chordino. Nature 387:862-863. CrossRef Medline

Shimizu T, Bae YK, Muraoka O, Hibi M (2005) Interaction of Wnt and caudal-related genes in zebrafish posterior body formation. Dev Biol 279: 125-141. CrossRef Medline

Sirotkin HI, Dougan ST, Schier AF, Talbot WS (2000) bozozok and squint act in parallel to specify dorsal mesoderm and anterior neuroectoderm in zebrafish. Development 127:2583-2592. Medline

Stern CD (2006) Neural induction: 10 years on since the 'default model.' Curr Opin Cell Biol 18:692-697. CrossRef Medline

Sun Z, Jin P, Tian T, Gu Y, Chen YG, Meng A (2006) Activation and roles of ALK4/ALK7-mediated maternal TGFbeta signals in zebrafish embryo. Biochem Biophys Res Commun 345:694-703. CrossRef Medline

Thisse B, Wright CV, Thisse C (2000) Activin- and Nodal-related factors control antero-posterior patterning of the zebrafish embryo. Nature 403: 425-428. CrossRef Medline

Tian T, Meng AM (2006) Nodal signals pattern vertebrate embryos. Cell Mol Life Sci 63:672-685. CrossRef Medline

Tsukiyama T, Wu C (1995) Purification and properties of an ATP-dependent nucleosome remodeling factor. Cell 83:1011-1020. CrossRef Medline

Woo K, Fraser SE (1997) Specification of the zebrafish nervous system by nonaxial signals. Science 277:254-257. CrossRef Medline

Woo K, Fraser SE (1998) Specification of the hindbrain fate in the zebrafish. Dev Biol 197:283-296. CrossRef Medline

Wu MY, Hill CS (2009) Tgf-beta superfamily signaling in embryonic development and homeostasis. Dev Cell 16:329-343. CrossRef Medline

Wysocka J, Swigut T, Xiao H, Milne TA, Kwon SY, Landry J, Kauer M, Tackett AJ, Chait BT, Badenhorst P, Wu C, Allis CD (2006) A PHD finger of NURF couples histone $\mathrm{H} 3$ lysine 4 trimethylation with chromatin remodelling. Nature 442:86-90. CrossRef Medline

Xiao H, Sandaltzopoulos R, Wang HM, Hamiche A, Ranallo R, Lee KM, Fu D, Wu C (2001) Dual functions of largest NURF subunit NURF301 in nucleosome sliding and transcription factor interactions. Mol Cell 8:531543. CrossRef Medline

Xi Q, He W, Zhang XH, Le HV, Massagué J (2008) Genome-wide impact of the BRG1 SWI/SNF chromatin remodeler on the transforming growth factor beta transcriptional program. J Biol Chem 283:1146-1155. CrossRef Medline

Xi Q, Wang Z, Zaromytidou AI, Zhang XH, Chow-Tsang LF, Liu JX, Kim H, Barlas A, Manova-Todorova K, Kaartinen V, Studer L, Mark W, Patel DJ, Massagué J (2011) A poised chromatin platform for TGF-beta access to master regulators. Cell 147:1511-1524. CrossRef Medline

Zeng L, Zhou MM (2002) Bromodomain: an acetyl-lysine binding domain. FEBS Lett 513:124-128. CrossRef Medline

Zhou X, Sasaki H, Lowe L, Hogan BL, Kuehn MR (1993) Nodal is a novel TGF-beta-like gene expressed in the mouse node during gastrulation. Nature 361:543-547. CrossRef Medline 